\title{
Crop Level and Harvest Date Impact on Four Ontario Wine Grape Cultivars. II. Wine Aroma Compounds and Sensory Analysis
}

\author{
L.H. Morena Luna ${ }^{1}$, A.G. Reynolds ${ }^{1 *}$, F.A. di Profio ${ }^{1}$, L. Zhang ${ }^{2}$, E. Kotsaki ${ }^{1}$ \\ (1) Cool Climate Oenology and Viticulture Institute, Brock University, St. Catharines, Ontario, Canada \\ (2) Geisenheim University, Geisenheim, Germany
}

Submitted for publication: January 2018

Accepted for publication: June 2018

Keywords: Crop reduction, harvest date, vintage effect, gas chromatography/mass spectrometry, wine aroma composition

\begin{abstract}
Pinot gris, Riesling, Cabernet franc and Cabernet Sauvignon from a vineyard in Virgil, Ontario, Canada were subjected by cluster thinning to two crop-level treatments (full crop, half crop), combined with three harvest dates (commercial harvest (T0), three weeks after T0 (T1), six weeks after T0 (T2)) in a randomised experiment in 2011 and 2012, with wines produced from all treatment replicates. We hypothesised that a full crop with substantially delayed harvest date might have a greater impact on wine aroma than reducing crop level. Wine aroma volatile analysis was carried out by GC-MS, with quantification by calibration with analytical standards prepared in model wine. Delayed harvest overcame the effects of crop reduction for almost all volatiles. Pinot gris and Riesling displayed increases in varietal aromas in the T1 and T2 wines, e.g. monoterpenes and norisoprenoids, and in esters, aldehydes and alcohols. Reduced concentrations of volatile acids and green odour compounds (e.g. 1-hexanol) with delayed harvest were also evident. Increases in ethanol were related to increased berry sugars, but higher alcohols did not necessarily increase with harvest date. Crop level had little impact on the sensory properties of these cultivars in both seasons. However, delayed harvest resulted in substantial sensorial changes. Due to these chemical and sensorial effects, delayed harvest is recommended, rather than crop-level reduction, to enhance wine quality in these cultivars. Extended harvest, e.g. T2 treatments, was associated with the production of benzaldehyde, diethyl acetal, and higher concentrations of higher alcohols, e.g. isoamyl alcohol and nonanol, which could be linked to pre-harvest desiccation.
\end{abstract}

\section{INTRODUCTION}

In wine, more than 800 compounds have been identified in their volatile fraction (Robinson et al., 2014), although a small subset of these are significant in terms of odour activity. Some of these compounds can be associated with varietal characteristics or are generated during fermentation, while others are considered undesirable when they occur (Bakker $\&$ Clarke, 2012). Non-volatile grape-derived precursors such as glycosides release monoterpenes, norisoprenoids and some thiols through enzymatic action by bacteria and yeasts, while other secondary metabolites, such as esters, volatile acids, higher alcohols and diacetyl, are likewise synthesised from the action of yeasts and malolactic bacteria (Schwab et al., 2008; Costantini et al., 2009).

Viticultural practices are highly influential in berry and wine composition. The effects of crop-level reduction on berry composition are normally an increase in soluble solids (Brix) and a concomitant increase in ethanol in the wine produced. Crop reduction may increase concentrations of free and bound terpenes (Reynolds et al., 2007), individual monoterpenes and norisoprenoids (Meyers et al., 2013), anthocyanins and phenols (King et al., 2012), as well as volatile acids (Bravdo et al., 1984). A delay of harvest is also linked to an increase in Brix by a reduction in berry weight due to dehydration processes (Costantini et al., 2006; Chkaiban et al., 2007). In addition to Brix, phenolics (Casassa et al., 2013) and aroma compounds (Bellincontro et al., 2004; Costantini et al., 2006) are either concentrated or new ones are produced. The drying of fruit also generates shrinkage, which modifies the shape and dimension of molecules, thus affecting mass transport phenomena (Kays, 1997). With a loss of water, cell wall enzyme activity is increased, and respiration and ethylene production are accelerated (Bellincontro et al., 2004; Costantini et al., 2006). This change or reduction in volatiles and polyphenols is not only due to concentration, but also to changes in metabolism (Bellincontro et al., 2004; Costantini et al., 2006; Zamboni et al., 2008). Dehydration by controlled processes reduces ethyl acetate and acetic acid (Moreno et al., 2008), increases ethanol and acetaldehyde, and gives rise to a reduction in alcohol dehydrogenase and proline (Zamboni et al., 2008).

*Corresponding author: E-mail address: areynolds@brocku.ca [Tel.: +905-688-5550 ext. 3131; Fax: +905-688-3104]

Acknowledgments: Our thanks are extended to Pondview Estate Winery, for their cooperation, and to the Ontario Research Fund, for financial support 
Wines made from dehydrated grapes may contain more terpenes and norisoprenoids (Moreno et al., 2008), but dehydration can lead to a decline in carotenoids, which are norisoprenoid precursors (Chkaiban et al., 2007). Grapes that undergo dehydration are susceptible to microbial spoilage, increased production of volatile acidity, Botrytis cinereaderived increases in polyalcohols, and the production of high amounts of other alcohols, such as glycerol, arabitol and mannitol (Sponholz, 1993). Sour rot reduces free geraniol, nerol and linalool concentrations and increases trans-furan linalool oxide, benzyl alcohol and 2-phenylethanol in Riesling (Zoecklein et al., 2010).

There has been an increased interest in delayed harvest in the region for the purpose of reducing vegetal characteristics and increasing the intensity of ripe fruit aromas. It was decided to experiment with different harvest dates to determine whether keeping a full crop with an extended harvest date might have a greater positive organoleptic impact and lead to larger increases in important odour-impact compounds than to reduce the crop level. The overall objective of this project was to determine the impact of harvest date and crop control on the wine volatile composition of four grape cultivars - two white wines (Riesling and Pinot gris) and two red wines (Cabernet Sauvignon and Cabernet franc) which are commonly produced in the Niagara Peninsula, Ontario, Canada. A secondary objective was to quantify total monoterpenes in Riesling must using a $\beta$-glycosidase enzyme treatment.

\section{MATERIALS AND METHODS}

\section{Experimental design}

All details of the experimental design are described in Moreno Luna et al. (2017). All trials were carried out at Pondview Estate Winery in the Four Mile Creek subappellation in Virgil, Ontario, Canada. Two crop levels imposed at véraison, viz. full crop (FC) and half crop (HC), and three harvest dates (including two harvest dates after regular harvest), were combined in a randomised complete block with a factorial treatment arrangement containing six treatment combinations. Each cultivar (Pinot gris, Riesling, Cabernet franc, Cabernet Sauvignon) was divided into six blocks (replicates), and each block comprised of one row containing six, six-vine panels (treatment replicates). $\mathrm{FC}$ vines were non-cluster thinned, and $\mathrm{HC}$ vines were cluster thinned to one basal cluster per shoot. Harvest date treatments were T0 (normal commercial harvest), T1 (three weeks after T0), and T2 (six weeks after T0). Vines were trained to double Guyot with vertical shoot positioning, with clean cultivation/rye cover crop in alternate rows. Spacing (row x vine; m) was 2.8 x 0.9 (Riesling) and $2.8 \times 1.2$ (all others). Rootstocks were SO4 (Riesling) and Couderc 3309 (others). Year of planting was 2004 (Riesling) and 2000 (others). Clones were ENTAV 53 (Pinot gris), 21B Weis (Riesling), ENTAV 214 (Cabernet franc) and unknown (Cabernet Sauvignon).

Data were collected on: yield components, vine size, berry, must and wine composition (Moreno Luna et al., 2017); winter hardiness and cane carbohydrates (Lefebvre et al., 2015); and individual anthocyanins and phenolics (Black et al., 2016). Wines were produced from each field treatment replicate using standard methods (Reynolds et al., 2007; Di Profio et al., 2011; Moreno Luna et al., 2017). Fruit were carefully sorted prior to processing to eliminate the incorporation of any unsound fruit in the fermentations. The analyses of volatile compounds in the 2011 and 2012 wine samples by GC-MS and sensory descriptive analysis were performed to determine whether differences existed between the two crop levels and between harvest dates. Wines from adjacent field replicates (e.g. 1 and 2; 3 and 4; 5 and 6) were combined at bottling to produce three replicates for sensory analysis.

\section{GC-MS sample preparation}

Aroma analysis by GC-MS was carried out in the 2011 and 2012 wines for the four grape cultivars based on Bowen and Reynolds (2012), with adjustments. A $30 \mathrm{~mL}$ sample was taken from each wine treatment replicate immediately prior to bottling, and was kept at $4^{\circ} \mathrm{C}$ in the presence of $\mathrm{N}_{2}$ inert gas until analysis. In duplicate, $100 \mu \mathrm{L}$ of an internal standard, prepared with $10 \mu \mathrm{L}$ of $98 \%$ 1-dodecanol (Aldrich; Oakville, ON) in $10 \mathrm{~mL}$ of $100 \%$ ethyl alcohol (Commercial Alcohols, Brampton, ON), was poured into a $10 \mathrm{~mL}$ volumetric flask, followed by the addition of wine to the mark and mixed. The prepared sample was transferred into a $10 \mathrm{~mL}$ Gerstel extraction vial. A $10 \mathrm{~mm}$ stir bar ("Twister"; Gerstel, Baltimore, MD), coated with polydimethylsiloxane ( $0.5 \mathrm{~mm}$ film thickness), was added to the sample and it was stirred for $1 \mathrm{hr}$ at $1000 \mathrm{~g}$ for extraction at room temperature. After extraction. the stir bar was removed, rinsed with Milli-Q water (Millipore, Bedford, MA), and dried out with lint-free tissue, then placed in a $4 \mathrm{~mL}$ amber vial at $4^{\circ} \mathrm{C}$ until analysis on the same day. The stir bar was then inserted into the extraction glass tube inside the thermal desorption unit (TDU) attached to GC-MS.

\section{GC-MS conditions, and conditioning of material}

An Agilent 6890N/5975B GC-MS equipped with a Gerstel TDU, cooled injection system, and programmable temperature vaporisation was used. Columns were: Agilent 19091S-433 HP-5MS 5\% phenyl methylsiloxane, nominal length $30.0 \mathrm{~m}$, nominal diameter $250.00 \mu \mathrm{m}$, nominal film thickness $0.25 \mu \mathrm{m}$; J\&W 122-7032 DB-WAX nominal length $30.0 \mathrm{~m}$, nominal diameter $250.00 \mu \mathrm{m}$, nominal film thickness $0.25 \mu \mathrm{m}$. Instrument conditions were identical to those in Bowen and Reynolds (2012). MS information: Solvent delay: 3 min, SCAN acquisition method for identification of compounds, low mass: 30, high mass: 400, threshold: 150, and SIM/SCAN mode for quantification of aromatic compounds. Stir bars used for extraction were previously conditioned every time before use to avoid any cross-contamination. After analysis, each stir bar was kept overnight in a solution of 80:20 acetonitrile/methanol, allowed to dry, and then placed at $250^{\circ} \mathrm{C}$ for $2 \mathrm{~h}$ with a constant flow of $\mathrm{N}_{2}$ inert gas. All glass material was washed with Milli-Q water and methanol, and then dried at $250^{\circ} \mathrm{C}$ for $1 \mathrm{~h}$.

\section{Calibration compounds and odour activity values}

Scan analysis reflected the presence of more than 100 volatile compounds in wines from all cultivars. For calibration 
purposes, 31 compounds were chosen for quantification (Supplementary Table 1). Three-point calibration curves were created for each compound, consistent with the literature (Bowen \& Reynolds, 2012). Aromatic standards were obtained from: Acros Organics (Nepean, ON): $\beta$-citronellol, ethyl caprylate, geraniol, 1-heptanol and phenylethanol; Fisher Scientific (Nepean, ON): ethyl acetate and $\alpha$-terpineol; Fluka (Oakville, ON): diethyl succinate, linalool, 1-nonanol, 2-phenethyl acetate and terpinolene ( $\delta$-terpinene); Sigma-Aldrich (Oakville, ON): benzaldehyde, decanal, damascenone, decanoic acid, diethyl acetal, ethyl butyrate, ethyl caproate, ethyl decanoate, ethyl heptanoate, ethyl phenyl acetate, hexanoic acid, 1-hexanol, hexyl acetate, isoamyl acetate, isoamyl alcohol, isobutyl alcohol, octanoic acid and 1-octanol.

Model wine was used for calibration curves and prepared based on Bowen and Reynolds (2012), using 12\% (v/v) of pure anhydrous ethanol (Commercial Alcohols, Brampton, $\mathrm{ON}$ ) diluted in Milli-Q water and $5 \mathrm{~g} / \mathrm{L}$ of tartaric acid. The $\mathrm{pH}$ of the model wine was adjusted to 3.6 with $1 \mathrm{~N} \mathrm{NaOH}$. Each aroma standard was diluted first in pure anhydrous ethanol at $1000 \mathrm{mg} / \mathrm{L}$ and kept at $4^{\circ} \mathrm{C}$ until analysis, then diluted at different concentrations in model wine. Calibration samples were analysed in SIM/SCAN mode using the same conditions as described previously with the use of the same internal standard. Odour activity values (OAVs) were calculated as a ratio between each concentration obtained by calibration $v s$ their respective threshold. Thresholds were obtained from the literature (Buttery et al., 1968, 1982; Ruth, 1986; Buttery et al., 1988; Takeoka et al., 1990; Etiévant, 1991; Guth, 1997; Ferreira et al., 2000; Dunlevy et al., 2009).

\section{Analysis of Pinot gris and Riesling must}

The analysis of the must was based on Moio et al. (2004). Pinot gris and Riesling must samples from the 2011 and 2012 seasons, previously frozen at $-25^{\circ} \mathrm{C}$, were thawed and thereafter centrifuged using an IEC Centra CL2 for 10 $\min$ at $3500 \mathrm{~g}$. Samples were divided into two fractions: the first, without $\beta$-glucosidase added, was designated for the determination of free terpenes, while the second had $\beta$-glycosidase added to quantify total terpenes. Following centrifugation, the supernatant was recovered and the $\mathrm{pH}$ was adjusted to 5.0 with $20 \% \mathrm{NaOH}$. A solution containing a commercial enzyme product with $\beta$-glycosidase activity [LAFAZYM®AROM (Laffort, Bordeaux, France)] was prepared using $1.5 \mathrm{mg}$ in $7.5 \mathrm{~mL}$ of distilled water, and $1.5 \mathrm{~mL}$ of this solution was mixed with the must supernatant in accordance with the manufacturer's protocols (Laffort, 2013). The fraction designated for the measurement of free terpenes had $1.5 \mathrm{~mL}$ distilled water added. The mix was incubated at $40^{\circ} \mathrm{C}$ for $12 \mathrm{~h}$ under constant agitation to allow the reaction to proceed. During sample preparation, $\mathrm{N}_{2}$ gas was added to avoid oxidation of the aroma components. For both fractions, $10 \mathrm{~mL}$ of the prepared samples were analysed in duplicate in GC-MS. Identical procedures were used for the extraction of must aroma volatiles and their quantification as were used for the wines. However, model must was used for calibration curves and prepared using $100 \mathrm{~g} / \mathrm{L}$ each of glucose and fructose diluted in Milli-Q water and $5 \mathrm{~g} / \mathrm{L}$ of tartaric acid. The $\mathrm{pH}$ of the model must was adjusted to 3.6 with $1 \mathrm{~N} \mathrm{NaOH}$.

\section{Descriptive sensory analysis Panel training}

Both panel training and descriptive analysis were carried out consistent with the practices previously described for white (Reynolds et al., 2007) and red wines (Di Profio et al., 2011). The panel consisted of 12 panellists (six male and six female members) aged 23 to 56, and all participants were students or faculty members of the Brock University Cool Climate Oenology and Viticulture Institute (CCOVI) with previous tasting experience. Six 60 to 90 min training sessions were conducted weekly for each cultivar. Two wine samples, consisting of one T0 and one T1 or T2 treatment, were presented in each training session. In the first training session, tasters were asked to evaluate the wines based on a preliminary list of provided descriptors, and these descriptors were thereafter modified based on panel feedback to establish an agreed-upon vocabulary. A final list of aroma, flavour and basic taste descriptors was ultimately generated from the most frequently identified descriptors in the initial two sessions. Aroma standards were thereafter made as reference descriptors to define wine characteristics and were modified over the training period according to suggestions by the panel (Supplementary Table 2). All panellists were required to evaluate the standards to test for any possible anosmias; no panellists were dropped due to any apparent specific sensory inabilities based on the standards used. All standards were made up in advance of training in $500 \mathrm{~mL}$ of non-oaked, neutral Riesling and Cabernet franc base wines and stored at $4^{\circ} \mathrm{C}$. Standards were presented as $30 \mathrm{~mL}$ samples in ISO wine glasses; these represented the "high intensity" anchor term at the far right end of the respective line scales $(15 \mathrm{~cm})$. In each training session, panellists were required to independently evaluate the two wine samples and use an unstructured linear scale of 0 to 15 points to define the intensity of the wine attributes. Discussions took place among the panel when they finished their rating. Panel training occurred prior to sensory evaluation in October 2013 (Cabernet franc, Cabernet Sauvignon) and March 2014 (Riesling, Pinot gris).

\section{Descriptive analysis}

Tasting sessions were scheduled twice weekly in individual booths in the sensory lab at CCOVI. Compusense software (Version 5.0; Compusense, Guelph, ON) was used for data collection. Wines were kept at $18^{\circ} \mathrm{C}$ until tasting, and ISO glasses were used for the analysis. The aroma standards used in the training sessions were available to the panellists. A 1 min break between each sample and a 30 min break between each flight were provided. A 15-point linear scale, marked with "absent" and "high intensity" at each end, was used to indicate the intensity of aromas or flavours. Each panellist tasted one flight of six wines in duplicate for each of the three replicates (six flights in total), consisting of each crop level $\mathrm{x}$ harvest date treatment. For all cultivars, wines were marked with three-digit numbers and presented in a random order. Data collection took place from October to December 2013 
(Cabernet franc, Cabernet Sauvignon) and from April to June 2014 (Riesling, Pinot gris). In all cases, the 2011 wines were evaluated first. Each vintage was analysed separately, and all wines experienced $\sim 12$ months of bottle ageing prior to sensory evaluation; 2011 wines were bottled in July 2012, and the 2012 wines in May 2013. Wines had previously been stored in the CCOVI vinotheque at $12^{\circ} \mathrm{C}$.

\section{Statistical analysis}

The concentrations of all aroma compounds were analysed with SAS statistical software for analysis of variance to determine effects between crop-level treatments and harvest dates (PROC GLM; SAS Institute, Cary, NC). Duncan's multiple range test $(p \leq 0.05)$ was used to compare means. Sensory data were likewise analysed. Principal components analysis was used to elucidate further relationships among aroma compounds and sensory variables.

\section{RESULTS}

\section{Wine aroma compounds}

Pinot gris

A total of 23 volatile compounds were quantified (20 (2011) and 21 (2012) compounds are listed in Table 1) in the Pinot gris wines. Some were highly odour-active (see Supplementary Table 1 for thresholds) and could likely have generated an impact upon the aroma of the wines. Differences between treatments occurred for several compounds in 2011 and 2012 (Table 1). Only isobutyl alcohol, diethyl succinate and terpinolene (both detected only in the $\mathrm{HC}$ wines) differed between $\mathrm{HC}$ and FC in 2011. Isobutyl alcohol and citronellol were not detected in the 2012 wines. In 2012, hexyl acetate and terpinolene were reduced in the $\mathrm{HC}$ wines, ethyl phenyl

TABLE 1

Impact of harvest date and crop-level treatments on volatile compounds ( $\mu \mathrm{g} / \mathrm{L})$ of Ontario Pinot gris wines, Pondview Estate Winery, Virgil, ON, 2011 and 2012. Values are means of six replicates of each crop level x harvest date.

\begin{tabular}{|c|c|c|c|c|c|c|c|c|c|}
\hline \multirow[b]{2}{*}{ Compound } & \multicolumn{2}{|c|}{ Crop level } & \multirow[b]{2}{*}{$\begin{array}{l}\text { Signifi- } \\
\text { cance }^{a}\end{array}$} & \multicolumn{3}{|c|}{ Harvest date $^{b}$} & \multirow[b]{2}{*}{$\begin{array}{l}\text { Signifi- } \\
\text { cance }^{a}\end{array}$} & \multirow[b]{2}{*}{$\begin{array}{l}\text { Inter- } \\
\text { action }^{a}\end{array}$} & \multirow{2}{*}{$\begin{array}{l}\text { Mean } \\
\text { standard } \\
\text { deviation }\end{array}$} \\
\hline & Full & Half & & T0 ${ }^{\mathrm{c}}$ & T1 & $\mathbf{T 2}$ & & & \\
\hline \multicolumn{10}{|l|}{2011} \\
\hline \multicolumn{10}{|l|}{ Esters } \\
\hline Ethyl acetate & 207900 & 149100 & NS & 211800 & 177300 & 146300 & NS & NS & 7290 \\
\hline Ethyl butyrate & 170 & 140 & NS & 180 & 150 & 140 & NS & NS & 18.8 \\
\hline Ethyl caproate & 6600 & 7800 & NS & $7200 \mathrm{ab}$ & $10300 \mathrm{a}$ & $4100 b$ & $*$ & NS & 2210 \\
\hline Ethyl decanoate & 463 & 189 & NS & 599 & 270 & 110 & NS & NS & 320 \\
\hline Ethyl phenylacetate & 337 & 334 & NS & $\mathrm{ND}^{\mathrm{d}}$ & $506 a$ & $501 \mathrm{a}$ & $* * * *$ & NS & 5.0 \\
\hline 2-Phenethyl acetate & 11300 & 10900 & NS & 11400 & 11200 & 10700 & NS & NS & 430 \\
\hline Isoamyl acetate & 7500 & 7400 & NS & $7900 a$ & $8700 \mathrm{a}$ & $5800 b$ & $* *$ & NS & 1040 \\
\hline Hexyl acetate & 3900 & 3600 & NS & $5800 a$ & $5500 \mathrm{a}$ & ND & $* * * *$ & NS & 370 \\
\hline Diethyl succinate & ND & 3700 & $* * * *$ & $2900 \mathrm{a}$ & $2600 \mathrm{~b}$ & ND & $* * * *$ & $* * * *$ & 240 \\
\hline \multicolumn{10}{|l|}{ Alcohols } \\
\hline Isoamyl alcohol & 1431000 & 1411000 & NS & 1444000 & 1430000 & 1390000 & NS & NS & 133420 \\
\hline Isobutyl alcohol & 1234400 & 243400 & $* * * *$ & $1127500 \mathrm{a}$ & $742300 \mathrm{~b}$ & $346800 \mathrm{c}$ & $* * * *$ & $* * * *$ & 35260 \\
\hline Phenylethanol & 22600 & 16700 & NS & 24700 & 16500 & 17700 & NS & NS & 716 \\
\hline 1-Hexanol & 56900 & 55300 & NS & 58100 & 55400 & 55000 & NS & NS & 165 \\
\hline \multicolumn{10}{|l|}{$\underline{\text { Acids }}$} \\
\hline Hexanoic acid & 24100 & 11800 & NS & 30800 & 12700 & 10400 & NS & NS & 1723 \\
\hline Octanoic acid & 15900 & 15400 & NS & $17200 \mathrm{a}$ & $18900 \mathrm{a}$ & $10700 \mathrm{~b}$ & $* *$ & NS & 2960 \\
\hline Decanoic acid & 25400 & 25000 & NS & $27200 a$ & $36200 a$ & $12100 \mathrm{~b}$ & $* *$ & NS & 6230 \\
\hline \multicolumn{10}{|l|}{ Terpenes } \\
\hline Citronellol & 67 & 66 & NS & ND & ND & 199 & $* * * *$ & NS & 20 \\
\hline Terpinolene & ND & 298 & $* * * *$ & $156 \mathrm{a}$ & $150 \mathrm{~b}$ & $141 \mathrm{c}$ & $* * * *$ & $* * * *$ & 3.2 \\
\hline \multicolumn{10}{|l|}{ Norisoprenoid } \\
\hline$\beta$-Damascenone & 316 & 70 & NS & 358 & 131 & 90 & NS & NS & 190 \\
\hline \multicolumn{10}{|l|}{$\underline{\text { Other }}$} \\
\hline Diethyl acetal & 312500 & 313200 & NS & ND & ND & 938600 & $* * * *$ & NS & 2170 \\
\hline
\end{tabular}




\begin{tabular}{|c|c|c|c|c|c|c|c|c|c|}
\hline \multirow[b]{2}{*}{ Compound } & \multicolumn{2}{|c|}{ Crop level } & \multirow{2}{*}{$\begin{array}{l}\text { Signifi- } \\
\text { cance }^{a}\end{array}$} & \multicolumn{3}{|c|}{ Harvest date $^{b}$} & \multirow{2}{*}{$\begin{array}{l}\text { Signifi- } \\
\text { cance }^{a}\end{array}$} & \multirow{2}{*}{$\begin{array}{l}\text { Inter- } \\
\text { action }{ }^{a}\end{array}$} & \multirow{2}{*}{$\begin{array}{l}\text { Mean } \\
\text { standard } \\
\text { deviation }\end{array}$} \\
\hline & Full & Half & & T0 ${ }^{c}$ & $\mathbf{T 1}$ & T2 & & & \\
\hline \multicolumn{10}{|l|}{2012} \\
\hline \multicolumn{10}{|l|}{ Esters } \\
\hline Ethyl acetate & 207900 & 149100 & NS & 211800 & 177300 & 146300 & NS & NS & 16890 \\
\hline Ethyl butyrate & 310 & 190 & NS & $200 b$ & $180 \mathrm{~b}$ & $370 \mathrm{a}$ & $*$ & $*$ & 63 \\
\hline Ethyl caproate & 36100 & 9600 & NS & 12900 & 10800 & 44900 & NS & NS & 1398 \\
\hline Ethyl caprylate & 25100 & 25200 & NS & 28500 & 24000 & 22800 & NS & $*$ & 4060 \\
\hline Ethyl decanoate & 647 & 236 & NS & 217 & 196 & 912 & NS & NS & 53 \\
\hline Ethyl phenylacetate & 288 & ND & $* * *$ & ND & ND & 432 & $* * *$ & $* * *$ & 53 \\
\hline 2-Phenethyl acetate & 11900 & 10900 & NS & 11200 & 10900 & 12200 & NS & NS & 497 \\
\hline Isoamyl acetate & 19200 & 10700 & NS & 15900 & 9900 & 19000 & NS & NS & 4940 \\
\hline Hexyl acetate & 3900 & 2000 & $* * * *$ & $6100 \mathrm{a}$ & $2900 b$ & ND & $* * * *$ & $* * * *$ & 41 \\
\hline Diethyl succinate & 2900 & 1100 & NS & ND & ND & 6000 & $*$ & NS & 1590 \\
\hline \multicolumn{10}{|l|}{ Alcohols } \\
\hline Isoamyl alcohol & 3807000 & 1550000 & NS & $1520000 \mathrm{~b}$ & $1635000 \mathrm{~b}$ & $4880000 \mathrm{a}$ & $*$ & NS & 175410 \\
\hline Phenylethanol & 22400 & 14000 & NS & 15800 & 16200 & 25500 & NS & NS & 5830 \\
\hline 1-Hexanol & 58100 & ND & $* * * *$ & $27500 \mathrm{~b}$ & $27600 \mathrm{~b}$ & $32100 \mathrm{a}$ & $* * * *$ & $* * * *$ & 561 \\
\hline 1-Octanol & 229 & 213 & NS & 132 & 204 & 327 & NS & NS & 181 \\
\hline \multicolumn{10}{|l|}{$\underline{\text { Acids }}$} \\
\hline Hexanoic acid & 48600 & 17700 & NS & 19800 & 16000 & 63700 & NS & NS & 3100 \\
\hline Octanoic acid & 84400 & 25800 & NS & 37400 & 28900 & 98900 & NS & NS & 4280 \\
\hline Decanoic acid & 48500 & 43100 & NS & $64900 \mathrm{a}$ & $54400 b$ & $18200 \mathrm{c}$ & $* * * *$ & $*$ & 5630 \\
\hline \multicolumn{10}{|l|}{ Terpene } \\
\hline Terpinolene & 120 & 89 & $*$ & ND & ND & 314 & $* * * *$ & NS & 27 \\
\hline \multicolumn{10}{|l|}{ Norisoprenoid } \\
\hline \multicolumn{10}{|l|}{ Other } \\
\hline Diethyl acetal & 318300 & 313600 & NS & ND & ND & 948000 & $* * * *$ & NS & 2740 \\
\hline Benzaldehyde & 5500 & 900 & NS & ND & ND & 9600 & $*$ & NS & 2430 \\
\hline
\end{tabular}

a $* * * *, * * *, * * * *$, ns: Significant at $\mathrm{p} \leq 0.05,0.01,0.001,0.0001$ and not significant respectively.

${ }^{\mathrm{b}}$ Mean values with same letters are not significantly different at $\mathrm{p} \leq 0.05$, Duncan's multiple range test.

c T0, T1, T2: Normal commercial harvest, three weeks after T0 and six weeks after T0 respectively.

${ }^{\mathrm{d}} \mathrm{ND}=$ Not detected.

acetate and 1-hexanol were not detected in the $\mathrm{HC}$ wines, while $\beta$-damascenone increased.

More compounds were affected by harvest date than by crop reduction. Ethyl caproate was reduced by the extended harvest date in 2011, and tended to increase in 2012, and higher concentrations were detected at all harvest dates in 2012 compared to 2011. Ethyl phenyl acetate increased in both years relative to harvest date, while diethyl succinate decreased in 2011 and increased in 2012; both were not detected in the $2011 \mathrm{~T} 2$ wines and in the $2012 \mathrm{~T} 0$ and T1 wines. Hexyl acetate was apparently reduced with delayed harvest in both years, with undetected levels at T2. Isobutyl alcohol was reduced by delayed harvest in 2011. 1-Hexanol increased with delayed harvest in 2012, and concentrations were lower than in 2011. Octanoic acid was reduced with delayed harvest in 2011, but tended to increase slightly in 2012, while decanoic acid decreased with delayed harvest in both years. Citronellol was only detected at T2 in 2011. Terpinolene decreased with extended harvest date in 2011, but was only detected at T2 in 2012. Diethyl acetal was only detected at T2 in both years, as was benzaldehyde in 2012.

There were several crop level $\mathrm{x}$ harvest date interactions for some compounds, but many of these existed due to the non-detection of these compounds in specific treatment combinations (data not shown). This was also the case for the other cultivars. PCA clustered most treatments on the 
negative side of $\mathrm{PC} 1$, associated with decanoic acid and hexyl acetate, while the 2011 T2 wines were clustered in the lower left quadrant, associated with citronellol and isobutanol (Supplementary Fig. 1). One outlier (HC/T2 2012) was positioned in the lower right quadrant.

\section{Riesling}

A total of 27 volatile compounds were quantified in Riesling wines (24 (2011) and 23 (2012) compounds are listed in Table 2). Reduction in crop affected a few compounds
(Table 2). Ethyl butyrate, phenylethanol, citronellol, geraniol and $\beta$-damascenone were reduced in concentration by cluster thinning in 2012, but were not affected in 2011. 1-Hexanol and isobutyl alcohol were reduced by crop reduction in both years; the latter was not detected in the HC wines in 2012. Some compounds were only detected in one year. In 2011, diethyl acetal was only present in FC/T2, nonyl aldehyde was not detected in the $\mathrm{HC}$ wines, while geraniol increased with crop reduction. Hexyl acetate increased with crop reduction in 2011, but was reduced in 2012, and the same occurred for

TABLE 2

Impact of harvest date and crop level treatments on volatile compounds $(\mu \mathrm{g} / \mathrm{L})$ of Ontario Riesling wines, Pondview Estate Winery, Virgil, ON, 2011-2012. Values are means of six replicates of each crop level x harvest date.

\begin{tabular}{|c|c|c|c|c|c|c|c|c|c|}
\hline \multirow[b]{2}{*}{ Compound } & \multicolumn{2}{|c|}{ Crop level } & \multirow[b]{2}{*}{$\begin{array}{l}\text { Signif- } \\
\text { icance }^{a}\end{array}$} & \multicolumn{3}{|c|}{ Harvest date $^{b}$} & \multirow[b]{2}{*}{$\begin{array}{l}\text { Signif- } \\
\text { icance }^{a}\end{array}$} & \multirow[b]{2}{*}{$\begin{array}{l}\text { Inter- } \\
\text { action }^{a}\end{array}$} & \multirow{2}{*}{$\begin{array}{l}\text { Mean } \\
\text { standard } \\
\text { deviation }\end{array}$} \\
\hline & Full & Half & & T0 ${ }^{c}$ & T1 & $\mathbf{T} 2$ & & & \\
\hline \multicolumn{10}{|l|}{2011} \\
\hline \multicolumn{10}{|l|}{ Esters } \\
\hline Ethyl acetate & 169400 & 162800 & NS & $145400 \mathrm{~b}$ & $138700 \mathrm{~b}$ & $214300 \mathrm{a}$ & $*$ & NS & 28618 \\
\hline Ethyl butyrate & 150 & 150 & NS & 150 & 140 & 150 & NS & NS & 3.2 \\
\hline Ethyl caproate & 4600 & 4700 & NS & $6600 \mathrm{a}$ & $1800 \mathrm{~b}$ & $5400 \mathrm{a}$ & $* * *$ & * & 1168 \\
\hline Ethyl caprylate & 15900 & 16500 & NS & $24100 \mathrm{a}$ & $6900 \mathrm{~b}$ & $17600 \mathrm{a}$ & $* * *$ & $* *$ & 4617 \\
\hline Ethyl decanoate & 280 & 178 & NS & $223 \mathrm{ab}$ & $72 b$ & $394 a$ & $*$ & $\mathrm{NS}$ & 122 \\
\hline Ethyl phenylacetate & 510 & 490 & $*$ & 500 & 493 & 506 & NS & NS & 8.3 \\
\hline 2-Phenethyl acetate & 10700 & 10800 & NS & 10800 & 10700 & 10800 & NS & $\mathrm{NS}$ & 99 \\
\hline Isoamyl acetate & 6500 & 7100 & NS & $7700 \mathrm{a}$ & $5700 \mathrm{~b}$ & $7000 \mathrm{a}$ & $* *$ & NS & 777 \\
\hline Hexyl acetate & 3600 & 5500 & $* * * *$ & $5700 \mathrm{a}$ & $5300 \mathrm{~b}$ & $2600 \mathrm{c}$ & $* * * *$ & $* * * *$ & 120 \\
\hline \multicolumn{10}{|l|}{ Alcohols } \\
\hline Isoamyl alcohol & 1320000 & 1344500 & NS & 1316600 & 1233400 & 1446800 & NS & NS & 132106 \\
\hline Isobutyl alcohol & 268000 & 237000 & $*$ & $\mathrm{ND}^{\mathrm{d}}$ & ND & 757600 & $* * * *$ & $*$ & 55220 \\
\hline Phenylethanol & 17300 & 16900 & NS & 16700 & 16700 & 17900 & NS & NS & 902 \\
\hline 1-Hexanol & 55200 & 37000 & $* * * *$ & $55800 \mathrm{a}$ & $55200 \mathrm{~b}$ & $27300 c$ & $* * * *$ & $* * * *$ & 106 \\
\hline \multicolumn{10}{|l|}{ Acids } \\
\hline Hexanoic acid & 11300 & 12900 & NS & $14900 \mathrm{a}$ & $9900 b$ & $11600 b$ & $* *$ & $\mathrm{NS}$ & 1563 \\
\hline Octanoic acid & 17100 & 19600 & NS & $26000 \mathrm{a}$ & $11000 \mathrm{c}$ & $18000 b$ & $* * * *$ & $*$ & 2862 \\
\hline Decanoic acid & 29300 & 28400 & NS & $33800 \mathrm{a}$ & $12900 \mathrm{~b}$ & $39700 \mathrm{a}$ & $* * * *$ & $* *$ & 5697 \\
\hline \multicolumn{10}{|l|}{$\underline{\text { Terpenes }}$} \\
\hline Citronellol & 164 & 161 & NS & ND & $215 \mathrm{a}$ & $273 a$ & $* * *$ & $\mathrm{NS}$ & 87 \\
\hline Geraniol & 23 & 83 & $* * * *$ & $83 a$ & $41 b$ & $35 b$ & $* * * *$ & $* * * *$ & 16.7 \\
\hline Linalool & 570 & 620 & NS & $480 \mathrm{~b}$ & $610 \mathrm{ab}$ & $700 \mathrm{a}$ & $* * * *$ & NS & 126 \\
\hline$\alpha$-Terpineol & 1030 & 940 & NS & 950 & 910 & 1100 & NS & NS & 121 \\
\hline Terpinolene & 109 & 309 & $* * * *$ & $153 b$ & $158 b$ & $316 a$ & $* * * *$ & $* * * *$ & 19.5 \\
\hline \multicolumn{10}{|l|}{ Norisoprenoid } \\
\hline$\beta$-Damascenone & 102 & 92 & NS & $125 \mathrm{a}$ & $64 \mathrm{~b}$ & $101 \mathrm{ab}$ & $*$ & NS & 24.8 \\
\hline \multicolumn{10}{|l|}{ Other } \\
\hline Diethyl acetal & 314800 & ND & $* * * *$ & ND & $\mathrm{ND}$ & 472300 & $* * * *$ & $* * * *$ & 4965 \\
\hline Nonyl aldehyde & 144 & ND & $* * * *$ & ND & 217 & ND & $* * * *$ & $* * * *$ & 1.0 \\
\hline
\end{tabular}




\begin{tabular}{|c|c|c|c|c|c|c|c|c|c|}
\hline \multirow[b]{2}{*}{ Compound } & \multicolumn{2}{|c|}{ Crop level } & \multirow[b]{2}{*}{$\begin{array}{l}\text { Signif- } \\
\text { icance }^{\mathrm{a}}\end{array}$} & \multicolumn{3}{|c|}{ Harvest date $^{b}$} & \multirow[b]{2}{*}{$\begin{array}{l}\text { Signif- } \\
\text { icance }^{a}\end{array}$} & \multirow[b]{2}{*}{$\begin{array}{l}\text { Inter- } \\
\text { action }^{\text {a }}\end{array}$} & \multirow{2}{*}{$\begin{array}{l}\text { Mean } \\
\text { standard } \\
\text { deviation }\end{array}$} \\
\hline & Full & Half & & T0 ${ }^{c}$ & T1 & $\mathbf{T 2}$ & & & \\
\hline \multicolumn{10}{|l|}{2012} \\
\hline \multicolumn{10}{|l|}{ Esters } \\
\hline Ethyl acetate & 139200 & 145300 & NS & $155100 \mathrm{a}$ & $150200 \mathrm{a}$ & $121500 \mathrm{~b}$ & $* * * *$ & $*$ & 5983 \\
\hline Ethyl butyrate & 160 & 50 & $* * * *$ & $80 \mathrm{~b}$ & $90 b$ & $160 \mathrm{a}$ & $* * * *$ & $* * * *$ & 7.1 \\
\hline Ethyl caproate & 8100 & 7500 & NS & 8800 & 8100 & 6600 & NS & NS & 2237 \\
\hline Ethyl caprylate & 12800 & 11300 & NS & $13700 \mathrm{a}$ & $12300 \mathrm{ab}$ & $10100 \mathrm{~b}$ & $*$ & NS & 2091 \\
\hline Ethyl heptanoate & 88 & 77 & NS & ND & ND & 248 & $* * * *$ & NS & 56 \\
\hline Ethyl decanoate & 92 & 71 & NS & 94 & 85 & 66 & NS & NS & 17.7 \\
\hline Ethyl phenylacetate & ND & 160 & $* * * *$ & ND & ND & 242 & $* * * *$ & $* * * *$ & 7.5 \\
\hline 2-Phenethyl acetate & 11000 & 11000 & NS & $10800 b$ & $11100 \mathrm{a}$ & $11100 \mathrm{a}$ & $* * * *$ & NS & 64 \\
\hline Isoamyl acetate & 14400 & 11500 & $* * *$ & $11000 b$ & $11400 b$ & $16500 \mathrm{a}$ & $* * * *$ & $* * * *$ & 1034 \\
\hline Hexyl acetate & 5700 & 3800 & $* * * *$ & $5700 \mathrm{a}$ & $5600 \mathrm{a}$ & $2900 b$ & $* * * *$ & $* * * *$ & 86 \\
\hline \multicolumn{10}{|l|}{$\underline{\text { Alcohols }}$} \\
\hline Isoamyl alcohol & 1286200 & 1268200 & NS & $1146900 \mathrm{~b}$ & $1413700 \mathrm{a}$ & $1271100 \mathrm{ab}$ & $*$ & NS & 138867 \\
\hline Isobutyl alcohol & 234000 & ND & $* * * *$ & ND & ND & 351400 & $* * * *$ & $* * * *$ & 11981 \\
\hline Phenylethanol & 15900 & 15500 & $* *$ & $14900 b$ & $16000 \mathrm{a}$ & $16200 \mathrm{a}$ & $* * * *$ & $*$ & 187 \\
\hline 1-Hexanol & 55300 & 18200 & $* * * *$ & $27800 b$ & $27600 b$ & $54900 \mathrm{a}$ & $* * * *$ & $* * * *$ & 313 \\
\hline 1-Heptanol & 570 & ND & $* * * *$ & ND & ND & 860 & $* * * *$ & $* * * *$ & 13.9 \\
\hline \multicolumn{10}{|l|}{$\underline{\text { Acids }}$} \\
\hline Hexanoic acid & 15900 & 14700 & NS & $18900 \mathrm{a}$ & $15300 b$ & $11700 \mathrm{c}$ & $* * *$ & NS & 1573 \\
\hline Octanoic acid & 29300 & 27400 & NS & $34700 \mathrm{a}$ & $30400 b$ & $19800 \mathrm{c}$ & $* * * *$ & $* *$ & 1956 \\
\hline Decanoic acid & 40300 & 42500 & NS & $56700 \mathrm{a}$ & $49600 \mathrm{a}$ & $17900 b$ & $* * *$ & $* *$ & 9324 \\
\hline \multicolumn{10}{|l|}{$\underline{\text { Terpenes }}$} \\
\hline Citronellol & 54 & ND & $* * * *$ & ND & ND & 82 & $* * * *$ & $* * * *$ & 31 \\
\hline Linalool & 370 & 300 & NS & ND & $\mathrm{ND}$ & 1000 & $* * * *$ & NS & 384 \\
\hline Terpinolene & 115 & 104 & $* * * *$ & ND & ND & 329 & $* * * *$ & $* * * *$ & 4.3 \\
\hline \multicolumn{10}{|l|}{ Norisoprenoid } \\
\hline$\beta$-Damascenone & 545 & 335 & $* *$ & $653 a$ & $416 b$ & $250 \mathrm{c}$ & $* * *$ & $*$ & 82 \\
\hline \multicolumn{10}{|l|}{$\underline{\text { Other }}$} \\
\hline Benzaldehyde & 449 & 140 & $* * *$ & ND & ND & 884 & $* * * *$ & $* * *$ & 185 \\
\hline
\end{tabular}

a. *,**,***,****,ns: Significant at $\mathrm{p} \leq 0.05,0.01,0.001,0.0001$, or not significant respectively.

${ }^{\mathrm{b}}$ Mean values with same letters are not significantly different at $\mathrm{p} \leq 0.05$, Duncan's multiple range test.

c T0, T1, T2: Normal commercial harvest, three weeks after T0, six weeks after T0, respectively.

${ }^{\mathrm{d}} \mathrm{ND}=$ Not detected.

isoamyl acetate and terpinolene. Ethyl phenyl acetate was reduced in the $\mathrm{HC}$ wines in 2011, but was detected only in the $\mathrm{HC}$ wines in 2012. Both 1-heptanol and citronellol were likewise undetected in the $2012 \mathrm{HC}$ wines.

Delayed harvest led to increases in ethyl butyrate (2012 only), and in isobutyl alcohol, terpinolene and citronellol (both years), and to decreases in hexyl acetate, octanoic acid, hexanoic acid, ethyl caprylate and $\beta$-damascenone in both years. 1-Hexanol and isoamyl acetate decreased with delayed harvest in 2011 but increased in 2012, while ethyl acetate and decanoic acid displayed the opposite trend. Some compounds were present only at one or two harvest dates in one of both years. Diethyl acetal was only present at $\mathrm{T} 2 / \mathrm{FC}$ in 2011, 1-heptanol at T2/FC in 2012, while geraniol decreased at all harvest dates, but only in 2011, nonyl aldehyde was present only in T1/FC wines, and ethyl heptanoate, ethyl phenylacetate, isobutyl alcohol, citronellol, linalool, terpinolene and benzaldehyde were only present in the T2 wines in 2012.

As with Pinot gris, there were some crop level $\mathrm{x}$ harvest 
date interactions for some compounds for specific treatment combinations (data not shown). PCA separated wines by vintage, with all 2011 wines in the lower right quadrant and the 2012 T0 and T1 wines in the lower left quadrant (Supplementary Fig. 2). The 2012 T2 wines were separated from the others and were associated with benzaldehyde, ethyl heptanoate and heptanoic acid.

\section{Cabernet franc}

In total, 22 volatile compounds were quantified in the Cabernet franc wines (Table 3). Crop reduction increased concentrations of ethyl phenyl acetate, isobutyl alcohol and citronellol in 2011, and in ethyl caproate, ethyl caprylate, ethyl phenylacetate, isoamyl acetate, 1 hexanol, 1-heptanol, citronellol and benzaldehyde in 2012, with reductions in ethyl heptanoate in both years (Table 3). In 2011, there were reductions in ethyl butyrate and ethyl heptanoate in the HC wines, while reductions occurred for ethyl heptanoate, diethyl succinate and decanal in 2012. 1-Heptanol and decanal were detected in 2012 only. Ethyl butyrate was the only compound in both years that decreased in the HC wines in 2011. Ethyl phenylacetate and citronellol were not detected in the FC wines in 2011, ethyl heptanoate was not detected in the HC wines in either season, and decanal was not detected in the $\mathrm{HC}$ wines in 2012.

With respect to harvest date, diethyl acetal and diethyl succinate were present in the T2 wines only in both years, while 1-hexanol, citronellol, $\beta$-damascenone and decanoic acid decreased. Compounds affected in 2011 relative to extended harvest date were ethyl acetate, ethyl butyrate, ethyl heptanoate, ethyl caprylate and benzaldehyde, which increased, and ethyl caproate, isoamyl alcohol, isobutyl alcohol, phenylethanol, 1-hexanol, octanoic acid, decanoic acid, citronellol and $\beta$-damascenone, all of which decreased. Ethyl heptanoate, diethyl acetal and benzaldehyde were detected in the T2 wines only, while ethyl phenylacetate,

TABLE 3

Impact of harvest date and crop level treatments on volatile compounds $(\mu \mathrm{g} / \mathrm{L})$ of Ontario Cabernet franc wines, Pondview Estate Winery, Virgil, ON, 2011-2012. Values are means of six replications of each crop level x harvest date.

\begin{tabular}{|c|c|c|c|c|c|c|c|c|c|}
\hline \multirow[b]{2}{*}{ Compound } & \multicolumn{2}{|c|}{ Crop level } & \multirow{2}{*}{$\begin{array}{l}\text { Signif- } \\
\text { icance }^{a}\end{array}$} & \multicolumn{3}{|c|}{ Harvest date ${ }^{b}$} & \multirow{2}{*}{$\begin{array}{l}\text { Signif- } \\
\text { icance }^{a}\end{array}$} & \multirow{2}{*}{$\begin{array}{l}\text { Inter- } \\
\text { action }^{a}\end{array}$} & \multirow{2}{*}{$\begin{array}{l}\text { Mean } \\
\text { standard } \\
\text { deviation }\end{array}$} \\
\hline & Full & Half & & T0 ${ }^{\mathrm{c}}$ & $\mathbf{T 1}$ & $\mathbf{T} 2$ & & & \\
\hline \multicolumn{10}{|l|}{2011} \\
\hline \multicolumn{10}{|l|}{ Esters } \\
\hline Ethyl acetate & 223200 & 219700 & NS & $172300 \mathrm{c}$ & $223000 b$ & $269100 \mathrm{a}$ & $* * * *$ & $* * *$ & 17356 \\
\hline Ethyl butyrate & 50 & 14 & $* * * *$ & $70 \mathrm{~b}$ & $70 b$ & $140 \mathrm{a}$ & $* * * *$ & $* * * *$ & 1.3 \\
\hline Ethyl caproate & 1010 & 960 & NS & $920 \mathrm{ab}$ & $1118 \mathrm{a}$ & $850 \mathrm{~b}$ & $* * * *$ & NS & 192 \\
\hline Ethyl caprylate & 2800 & 2700 & NS & $1900 \mathrm{~b}$ & $2900 \mathrm{ab}$ & 3400 & $*$ & NS & 923 \\
\hline Ethyl heptanoate & 292 & $\mathrm{ND}^{\mathrm{d}}$ & $* * * *$ & ND & ND & 438 & $* * * *$ & $* * * *$ & 50 \\
\hline Ethyl decanoate & 24 & 23 & NS & $17 b$ & $31 \mathrm{a}$ & $22 \mathrm{ab}$ & $* * * *$ & NS & 8.1 \\
\hline Ethyl phenylacetate & ND & 164 & $* * * *$ & 246 & ND & ND & $* * * *$ & $* * * *$ & 6.4 \\
\hline 2-Phenethyl acetate & 10500 & 10500 & NS & 10500 & 10500 & 10600 & NS & NS & 9.4 \\
\hline Isoamyl acetate & 5000 & 5000 & NS & 5000 & 5000 & 5000 & NS & NS & 80 \\
\hline Diethyl succinate & 7200 & 6600 & NS & $6800 \mathrm{ab}$ & $5300 \mathrm{~b}$ & $8600 \mathrm{a}$ & $*$ & NS & 1239 \\
\hline \multicolumn{10}{|l|}{ Alcohols } \\
\hline Isoamyl alcohol & 1958800 & 1896500 & NS & $2056100 \mathrm{a}$ & $2030800 \mathrm{a}$ & $1596000 \mathrm{~b}$ & $*$ & NS & 262790 \\
\hline Isobutyl alcohol & 633000 & 740400 & $* * * *$ & $683300 \mathrm{~b}$ & $743700 \mathrm{a}$ & $633000 \mathrm{c}$ & $* * * *$ & $* * * *$ & 35947 \\
\hline Phenylethanol & 20100 & 21000 & NS & $21700 a$ & $21700 a$ & $18200 b$ & $* *$ & NS & 1492 \\
\hline 1-Hexanol & 37400 & 37400 & NS & $56400 \mathrm{a}$ & $55800 \mathrm{~b}$ & ND & $* * * *$ & NS & 458 \\
\hline \multicolumn{10}{|l|}{$\underline{\text { Acids }}$} \\
\hline Octanoic acid & 5500 & 5700 & NS & $5900 \mathrm{a}$ & $5800 \mathrm{a}$ & $5000 \mathrm{~b}$ & $* *$ & NS & 388 \\
\hline Decanoic acid & 2400 & 2800 & NS & $3500 \mathrm{a}$ & $3300 \mathrm{a}$ & $980 \mathrm{~b}$ & $* * *$ & NS & 676 \\
\hline \multicolumn{10}{|l|}{ Terpene } \\
\hline Citronellol & ND & 145 & $* * * *$ & $102 \mathrm{a}$ & $115 \mathrm{a}$ & ND & $* * *$ & $* * *$ & 67 \\
\hline \multicolumn{10}{|l|}{ Norisoprenoid } \\
\hline$\beta$-Damascenone & 103 & 146 & NS & $150 \mathrm{a}$ & $128 \mathrm{a}$ & $95 b$ & $* *$ & $*$ & 44 \\
\hline \multicolumn{10}{|l|}{$\underline{\text { Other }}$} \\
\hline Diethyl acetal & 314100 & 314500 & NS & ND & ND & 942900 & $* * * *$ & NS & 1930 \\
\hline Benzaldehyde & 557 & 579 & NS & ND & ND & 1700 & $* * * *$ & NS & 312 \\
\hline
\end{tabular}




\begin{tabular}{|c|c|c|c|c|c|c|c|c|c|}
\hline \multirow[b]{2}{*}{ Compound } & \multicolumn{2}{|c|}{ Crop level } & \multirow{2}{*}{$\begin{array}{l}\text { Signif- } \\
\text { icance }^{a}\end{array}$} & \multicolumn{3}{|c|}{ Harvest date $^{b}$} & \multirow{2}{*}{$\begin{array}{l}\text { Signif- } \\
\text { icance }^{\text {a }}\end{array}$} & \multirow{2}{*}{$\begin{array}{l}\text { Inter- } \\
\text { action }^{\mathrm{a}}\end{array}$} & \multirow{2}{*}{$\begin{array}{l}\text { Mean } \\
\text { standard } \\
\text { deviation }\end{array}$} \\
\hline & Full & Half & & T0 ${ }^{c}$ & T1 & $\mathbf{T} 2$ & & & \\
\hline \multicolumn{10}{|l|}{2012} \\
\hline \multicolumn{10}{|l|}{ Esters } \\
\hline Ethyl acetate & 122100 & 124800 & NS & 127800 & 123900 & 117000 & NS & NS & 10254 \\
\hline Ethyl butyrate & 130 & 130 & NS & 130 & 130 & 130 & NS & NS & 0.54 \\
\hline Ethyl caproate & 420 & 680 & $* * *$ & 590 & 510 & 530 & NS & NS & 60 \\
\hline Ethyl caprylate & 520 & 740 & $*$ & $500 \mathrm{~b}$ & $530 \mathrm{~b}$ & $980 \mathrm{a}$ & $*$ & NS & 192 \\
\hline Ethyl heptanoate & 15 & ND & $* * * *$ & ND & ND & 31 & $* * * *$ & $* * * *$ & 0 \\
\hline Ethyl decanoate & 14 & 21 & NS & $14 b$ & $16 b$ & $24 a$ & $* * * *$ & NS & 3.8 \\
\hline Ethyl phenylacetate & 123 & 183 & $* * * *$ & $244 b$ & ND & $246 a$ & $* * * *$ & $* * * *$ & 1.3 \\
\hline 2-Phenethyl acetate & 10500 & 10500 & NS & 10500 & 10500 & 10500 & NS & NS & 6.1 \\
\hline Isoamyl acetate & 4900 & 6100 & $*$ & $4900 \mathrm{~b}$ & $5000 \mathrm{~b}$ & $7200 \mathrm{a}$ & $* * *$ & $*$ & 717 \\
\hline Diethyl succinate & 7800 & 5600 & $*$ & $3400 \mathrm{c}$ & $9700 \mathrm{a}$ & $7200 b$ & $* * *$ & $*$ & 931 \\
\hline \multicolumn{10}{|l|}{$\underline{\text { Alcohols }}$} \\
\hline Isoamyl alcohol & 1210700 & 1357800 & NS & 1363600 & 1370300 & 1036100 & NS & NS & 356592 \\
\hline Isobutyl alcohol & 713000 & 689800 & NS & 712800 & 708500 & 691700 & NS & NS & 19276 \\
\hline Phenylethanol & 13100 & 13300 & NS & 13200 & 13300 & 13100 & NS & NS & 207 \\
\hline 1-Hexanol & 41400 & 41500 & $* *$ & $55700 \mathrm{a}$ & $54900 \mathrm{~b}$ & ND & $* * * *$ & $* * * *$ & 64 \\
\hline 1-Heptanol & 410 & 1040 & $* * * *$ & $840 \mathrm{~b}$ & ND & $1640 \mathrm{a}$ & $* * * *$ & $* * * *$ & 41 \\
\hline \multicolumn{10}{|l|}{$\underline{\text { Acids }}$} \\
\hline Octanoic acid & 5600 & 5900 & NS & 5800 & 6000 & 5200 & NS & NS & 410 \\
\hline Decanoic acid & 2700 & 3000 & NS & $3300 \mathrm{a}$ & $2700 \mathrm{~b}$ & $2400 b$ & $*$ & $* *$ & 356 \\
\hline \multicolumn{10}{|l|}{$\underline{\text { Terpene }}$} \\
\hline Citronellol & 67 & 190 & $*$ & $92 b$ & $250 \mathrm{a}$ & ND & $* *$ & NS & 95 \\
\hline \multicolumn{10}{|l|}{$\underline{\text { Norisoprenoid }}$} \\
\hline$\beta$-Damascenone & 156 & 104 & NS & $152 \mathrm{a}$ & $109 a$ & ND & $* * *$ & NS & 38 \\
\hline \multicolumn{10}{|l|}{ Other } \\
\hline Diethyl acetal & 233500 & 233300 & NS & ND & ND & 933500 & $* * * *$ & $* * * *$ & 101 \\
\hline Benzaldehyde & 4 & 29 & $* * * *$ & ND & ND & 66 & $* * * *$ & $* * * *$ & 0.71 \\
\hline Decanal & 112 & $\mathrm{ND}$ & $* * * *$ & ND & 149 & $\mathrm{ND}$ & $* * * *$ & $* * * *$ & 1.2 \\
\hline
\end{tabular}

a. $* * *, * * *, * * * *, n s:$ Significant at $\mathrm{p} \leq 0.05,0.01,0.001,0.0001$, or not significant respectively.

${ }^{\mathrm{b}}$ Mean values with same letters are not significantly different at $\mathrm{p} \leq 0.05$ by Duncan's multiple range test.

c T0, T1, T2: Normal commercial harvest, three weeks after T0, six weeks after T0, respectively.

${ }^{\mathrm{d}} \mathrm{ND}=$ Not detected.

1-hexanol and citronellol were not detected in the T2 wines. In 2012, ethyl caprylate, ethyl heptanoate, ethyl decanoate, ethyl phenyl acetate, isoamyl acetate, 1-heptanol and benzaldehyde increased relative to harvest date. 1-Hexanol, citronellol, $\beta$-damascenone and decanal were not detected in the T2 wines. Both 1-heptanol and decanal were only present in 2012; 1-heptanol at both crop levels and decanal in FC/T1. Ethyl heptanoate and benzaldehyde were only present in the T2 wines in both years.

As with the white wine cultivars, there were some crop level $\mathrm{x}$ harvest date interactions for some compounds for specific treatment combinations (data not shown). PCA once again separated wines by vintage, with the $2011 \mathrm{~T} 0$ and T1 wines grouped on the positive side of $\mathrm{PC} 2$, in or near the upper left quadrant, and the 2012 wines in the lower left quadrant (Supplementary Fig. 3). The T2 wines from both vintages were grouped in or near the lower right quadrant and associated with diethyl acetal, diethyl succinate, ethyl butyrate, heptanol and isoamyl acetate.

\section{Cabernet Sauvignon}

A total of 21 volatile compounds were quantified in the Cabernet Sauvignon wines, 18 (2011) and 20 (2012) of which are listed in Table 4. Crop reduction led to lower 1-hexanol concentrations and increased benzaldehyde in both years (Table 4). Hexanoic acid (2011) and ethyl heptanoate, diethyl succinate and 1-nonanol (2012) increased in the $\mathrm{HC}$ wines. Compounds that were reduced in the $\mathrm{HC}$ wines 
were 1-hexanol (both years); ethyl caproate, 2-phenylethyl acetate, isoamyl alcohol, phenylethanol, 1-nonanol, octanoic acid and decanoic acid (2011 only); and diethyl acetal (2012). Hexanoic acid was only present in HC/T0 in 2011, while it was reduced with crop reduction in 2012. 1-Nonanol, on the other hand, was only present in HC/T2 in 2012.

Delayed harvest led to reductions in 1-hexanol, phenylethanol, hexanoic acid, octanoic acid and decanoic acid in both years. $\beta$-Damascenone and diethyl acetal were reduced with delayed harvest (2011 only), while ethyl butyrate, ethyl caproate, isoamyl acetate and citronellol were reduced (2012). Hexanoic acid and diethyl acetal were not detected in the T2 wines (2011), while 2-phenylacetate, decanoic acid and citronellol were undetected in the 2012 T2 wines. Isobutyl alcohol and citronellol were only detected in the 2012 wines, with reductions relative to delayed harvest. Ethyl heptanoate was also present in 2012 only, with an increase between T1 and T2. Diethyl acetal was only present at T0 in 2011, and at FC/T2 in 2012. Benzaldehyde was only present at T2 in both years, and only at HC/T2 in 2011. $\beta$-Damascenone was only detected in the 2011 wines, with a reduction with delayed harvest date.

As with the other cultivars, there were crop level $\mathrm{x}$ harvest date interactions for several compounds for specific combinations (data not shown). PCA grouped the wines by vintage, but showed an obvious clustering of the T2 wines (Supplementary Fig. 4). The 2011 T0 and T1 wines were grouped in the upper right quadrant, and the 2012 wines were located in the upper left quadrant. The 2011 T2 wines were in the lower right quadrant and were associated with diethyl succinate, ethyl acetate and nonanol, whereas the 2012 T2 wines were in the lower left quadrant and associated primarily with benzaldehyde, ethyl butyrate and ethyl heptanoate.

\section{Analysis of Pinot gris and Riesling must}

Five monoterpenes were quantified in the 2011 Pinot gris and the 2011 and 2012 Riesling musts (Table 5). In the 2011 Pinot gris, there were no crop-level effects and nerol was highest in the T0 and T1 musts, while $\alpha$-terpineol was highest in the T2 musts. Despite the likelihood of some inhibition of $\beta$-glycosidase activity by glucose (Laffort,

TABLE 4

Impact of harvest date and crop level treatments on volatile compounds $(\mu \mathrm{g} / \mathrm{L})$ of Ontario Cabernet Sauvignon wines, Pondview Estate Winery, Virgil, ON, 2011-2012. Values are means of six replications of each crop level x harvest date.

\begin{tabular}{|c|c|c|c|c|c|c|c|c|c|}
\hline \multirow[b]{2}{*}{ Compound } & \multicolumn{2}{|c|}{ Crop level } & \multirow[b]{2}{*}{$\begin{array}{l}\text { Signif- } \\
\text { icance }^{\text {a }}\end{array}$} & \multicolumn{3}{|c|}{ Harvest date $^{b}$} & \multirow[b]{2}{*}{$\begin{array}{l}\text { Signif- } \\
\text { icance }^{\text {a }}\end{array}$} & \multirow[b]{2}{*}{$\begin{array}{l}\text { Inter- } \\
\text { action }^{\text {a }}\end{array}$} & \multirow{2}{*}{$\begin{array}{l}\text { Mean } \\
\text { standard } \\
\text { deviation }\end{array}$} \\
\hline & Full & Half & & T0 ${ }^{c}$ & T1 & $\mathbf{T 2}$ & & & \\
\hline \multicolumn{10}{|l|}{2011} \\
\hline \multicolumn{10}{|l|}{ Esters } \\
\hline Ethyl acetate & 486900 & 351100 & NS & $263300 \mathrm{c}$ & $407900 \mathrm{~b}$ & $585700 \mathrm{a}$ & $*$ & NS & 62200 \\
\hline Ethyl butyrate & 50 & 50 & NS & $\mathrm{ND}^{\mathrm{d}}$ & ND & 150 & $* * * *$ & NS & 5.2 \\
\hline Ethyl caproate & 3000 & 2100 & $*$ & 2200 & 2900 & 2500 & NS & NS & 579 \\
\hline Ethyl caprylate & 14000 & 6800 & NS & 13600 & 7500 & 10100 & NS & NS & 1253 \\
\hline Ethyl decanoate & 271 & 68 & NS & 340 & 74 & 94 & NS & NS & 188 \\
\hline 2-Phenethyl acetate & 10700 & 10600 & $* *$ & $10600 \mathrm{~b}$ & $10600 \mathrm{~b}$ & $10700 \mathrm{a}$ & $*$ & NS & 25 \\
\hline Isoamyl acetate & 8900 & 5300 & NS & 10500 & 5200 & 5500 & NS & NS & 121 \\
\hline $\begin{array}{l}\text { Diethyl succinate } \\
\underline{\text { Alcohols }}\end{array}$ & 15700 & 12800 & NS & $22300 \mathrm{a}$ & $7000 \mathrm{~b}$ & $13600 \mathrm{ab}$ & $*$ & NS & 5914 \\
\hline Isoamyl alcohol & 4339000 & 3463300 & $*$ & 3465300 & 4452900 & 3785200 & NS & NS & 631926 \\
\hline Phenylethanol & 34500 & 29700 & $* * *$ & $31400 \mathrm{~b}$ & $36200 a$ & $28700 \mathrm{c}$ & $* * *$ & $*$ & 2345 \\
\hline 1-Hexanol & 60000 & 58600 & $* *$ & $60900 \mathrm{a}$ & $59200 b$ & $57800 \mathrm{c}$ & $* * * *$ & $* *$ & 895 \\
\hline $\begin{array}{l}\text { 1-Nonanol } \\
\underline{\text { Acids }}\end{array}$ & 590 & 130 & $* * * *$ & $250 \mathrm{c}$ & $310 b$ & $510 \mathrm{a}$ & $* * * *$ & $* * * *$ & 42 \\
\hline Hexanoic acid & ND & 3400 & $* * * *$ & 5100 & ND & ND & $* * * *$ & $* * * *$ & 850 \\
\hline Octanoic acid & 7400 & 6500 & $* *$ & $7800 \mathrm{a}$ & $7000 \mathrm{~b}$ & $5900 \mathrm{c}$ & $* * *$ & $*$ & 411 \\
\hline $\begin{array}{l}\text { Decanoic acid } \\
\text { Norisoprenoid } \\
\end{array}$ & 6300 & 4700 & $* *$ & $7100 \mathrm{a}$ & $6600 \mathrm{a}$ & $2800 \mathrm{~b}$ & $* * * *$ & NS & 822 \\
\hline $\begin{array}{l}\beta \text {-Damascenone } \\
\text { Other }\end{array}$ & 697 & 57 & NS & $1026 a$ & $56 b$ & $46 b$ & $* *$ & NS & 16.0 \\
\hline Diethyl acetal & 623800 & 317700 & NS & 1412100 & ND & ND & $* * * *$ & NS & 5017 \\
\hline Benzaldehyde & ND & 1520 & $* * * *$ & ND & ND & 2280 & $* * * *$ & $* * * *$ & 1325 \\
\hline
\end{tabular}




\begin{tabular}{|c|c|c|c|c|c|c|c|c|c|}
\hline \multirow[b]{2}{*}{ Compound } & \multicolumn{2}{|c|}{ Crop level } & \multirow[b]{2}{*}{$\begin{array}{l}\text { Signif- } \\
\text { icance }\end{array}$} & \multicolumn{3}{|c|}{ Harvest date $^{b}$} & \multirow[b]{2}{*}{$\begin{array}{l}\text { Signif- } \\
\text { icance }^{\text {a }}\end{array}$} & \multirow[b]{2}{*}{$\begin{array}{l}\text { Inter- } \\
\text { action }^{\text {a }}\end{array}$} & \multirow{2}{*}{$\begin{array}{l}\text { Mean } \\
\text { standard } \\
\text { deviation }\end{array}$} \\
\hline & Full & Half & & To ${ }^{\mathrm{c}}$ & T1 & $\mathbf{T} 2$ & & & \\
\hline \multicolumn{10}{|l|}{2012} \\
\hline \multicolumn{10}{|l|}{ Esters } \\
\hline Ethyl acetate & 131300 & 130800 & NS & $125200 \mathrm{~b}$ & $140900 \mathrm{a}$ & $125100 \mathrm{~b}$ & $*$ & $* * *$ & 3986 \\
\hline Ethyl butyrate & 130 & 130 & NS & $133 a$ & $132 b$ & $131 \mathrm{~b}$ & $* *$ & $* *$ & 0.37 \\
\hline Ethyl caproate & 660 & 510 & NS & $750 \mathrm{a}$ & $650 \mathrm{a}$ & $240 \mathrm{~b}$ & $* *$ & NS & 125 \\
\hline Ethyl caprylate & 1100 & 1200 & NS & 1100 & 850 & 1600 & NS & NS & 421 \\
\hline Ethyl heptanoate & 1.3 & 6.4 & $* *$ & ND & $3.8 b$ & $9.8 \mathrm{a}$ & $* *$ & NS & 4.0 \\
\hline Ethyl decanoate & 29 & 24 & NS & $17 b$ & $28 \mathrm{ab}$ & $39 a$ & $*$ & NS & 9.5 \\
\hline 2-Phenethyl acetate & 7800 & 7800 & NS & $10400 \mathrm{a}$ & $10400 \mathrm{a}$ & ND & $* * * *$ & NS & 0 \\
\hline Isoamyl acetate & 4900 & 5000 & NS & $5100 \mathrm{a}$ & 4900ab & $4800 \mathrm{~b}$ & $*$ & NS & 106 \\
\hline Diethyl succinate & 5100 & 11500 & $*$ & $6100 \mathrm{~b}$ & $3400 \mathrm{~b}$ & $19000 \mathrm{a}$ & $*$ & $*$ & 675 \\
\hline \multicolumn{10}{|l|}{$\underline{\text { Alcohols }}$} \\
\hline Isoamyl alcohol & 645900 & 530000 & NS & $323400 \mathrm{~b}$ & $816500 \mathrm{a}$ & $641900 \mathrm{ab}$ & $*$ & $*$ & 2384 \\
\hline Isobutyl alcohol & 706400 & 717300 & NS & $747400 \mathrm{a}$ & $692800 \mathrm{~b}$ & $687000 \mathrm{~b}$ & $* *$ & $* * *$ & 18616 \\
\hline Phenylethanol & 21900 & 22500 & NS & $24400 \mathrm{a}$ & $21800 b$ & $19400 \mathrm{c}$ & $* * *$ & NS & 815 \\
\hline 1-Hexanol & 56000 & 55700 & * & $56300 \mathrm{a}$ & $55900 \mathrm{a}$ & $55300 \mathrm{~b}$ & $* *$ & $* *$ & 233 \\
\hline 1-Nonanol & ND & 45 & $* * * *$ & ND & ND & 90 & $* * * *$ & $* * * *$ & 5.6 \\
\hline \multicolumn{10}{|l|}{$\underline{\text { Acids }}$} \\
\hline Hexanoic acid & 8600 & 5800 & $* * * *$ & $8000 \mathrm{a}$ & $8000 \mathrm{a}$ & $4700 \mathrm{~b}$ & $* * * *$ & $* * * *$ & 250 \\
\hline Octanoic acid & 5600 & 5700 & NS & $5800 \mathrm{a}$ & $5800 \mathrm{a}$ & $5000 \mathrm{~b}$ & $* *$ & NS & 391 \\
\hline Decanoic acid & 3300 & 2600 & NS & $3300 \mathrm{a}$ & $4500 \mathrm{a}$ & ND & $* *$ & NS & 1199 \\
\hline \multicolumn{10}{|l|}{ Terpenes } \\
\hline Citronellol & 107 & 119 & NS & $182 \mathrm{a}$ & $119 b$ & ND & $* * * *$ & NS & 25 \\
\hline \multicolumn{10}{|l|}{ Other } \\
\hline Diethyl acetal & 233700 & ND & $* * * *$ & ND & ND & 467300 & $* * * *$ & $* * * *$ & 552 \\
\hline Benzaldehyde & 247 & 329 & $*$ & ND & ND & 1150 & $* * * *$ & $* *$ & 186 \\
\hline
\end{tabular}

a. ***,***,***,ns: Significant at $\mathrm{p} \leq 0.05,0.01,0.001,0.0001$, or not significant respectively.

${ }^{b}$ Mean values with same letters are not significantly different at $\mathrm{p} \leq 0.05$ by Duncan's multiple range test.

${ }^{\text {c }}$ T0, T1, T2: Normal commercial harvest, three weeks after T0, six weeks after T0, respectively.

${ }^{\mathrm{d}} \mathrm{ND}=$ Not detected.

2013), enzyme treatment increased the concentrations of geraniol, nerol and $\alpha$-terpineol. In the 2011 Riesling, there were no crop-level effects; terpinolene was highest in the T0 musts, while enzyme treatment increased linalool, nerol and $\alpha$-terpineol (Table 5). In the 2012 Riesling, reducing the crop level increased linalool only, delayed harvest (T2) increased all terpenes, while the use of enzyme increased linalool, geraniol and $\alpha$-terpineol, suggesting that small proportions of must nerol and hotrienol were glycosylated (Table 5).

\section{Sensory analysis}

\section{Main effects: Pinot gris}

Crop level had few effects on Pinot gris in both seasons (Supplementary Table 3). Reducing crop level increased body in 2011; in 2012, crop reduction led to lower lemon and bread flavours and acidity. Harvest date was responsible for several differences, including reductions in lemon (2011) and bread aroma (2012), and increases in honey $(2011,2012)$ and floral (2012) aromas and flavours (Fig. 1; Supplementary Table 3). Delayed harvest also increased pear flavour (2012), in addition to body and length (both seasons).

\section{Riesling}

Crop level had no impact on Riesling aroma in either year, but crop reduction reduced apple/pear, peach/apricot, and honey flavours in 2011, in addition to decreasing perceived sweetness and increasing acidity (Supplementary Table 4). A slight decrease in acidity relative to crop reduction was measured in 2012. Numerous aroma and flavour attributes were affected by harvest date (Fig. 2; Supplementary Table 4). Delayed harvest increased peach/apricot (2011, 2012), mango (2011, 2012), honey (2011) and floral (2012) aromas, and decreased green apple (2011) and grassy (2011) aromas, while apple/pear was highest for the T1 wines (2011). 
Delayed harvest led to increased peach/apricot $(2011,2012)$, mango (2011, 2012), floral (2012), honey (2012) and vanilla flavours (2012), in addition to greater body and length (2011), and enhanced sweetness plus reduced acidity (2012). Sweetness was reduced and acidity increased by delayed harvest in 2011. Delayed harvest also increased apple/pear flavour in the T1 wines (2011).

\section{Cabernet franc}

A reduced crop led to reductions in red fruit aroma and flavour in 2011, but the response was opposite in the much warmer 2012 season (Supplementary Table 5). Crop reductions also reduced vegetal aroma and dried fruit flavour in 2012. Numerous harvest date-related responses occurred (Fig. 3; Supplementary Table 5); these included increases in dried fruit (2011), earthy (2011), red fruit (2012) and dark fruit (2012) aromas, and decreases in red and dark fruit (2011), herbaceous (2011), vegetal (2012) and earthy (2012) aromas. Flavour attributes that increased with delayed harvest included: dried fruit $(2011,2012)$, spicy (2011) and dark fruit (2012), while red fruit (2011) declined. Body (2011) likewise increased relative to delayed harvest, while astringency and acidity were highest in the T1 wines in 2011. Insufficient volumes were available for the T2 treatments in 2012 for sensory evaluation.

\section{Cabernet Sauvignon}

Few crop-level effects were measured, with the exception of increases in dark fruit flavour (2011), and reductions in astringency, bitterness and length (2012) (Supplementary Table 6). The majority of harvest-date effects were confined to 2011; delayed harvest increased dried fruit (2011), earthy (2011) and dark fruit (2012) aromas and reduced red and dark fruit (2011) and herbaceous (2011) aromas (Fig. 4; Supplementary Table 6). Delayed harvest also led to increases in dried fruit (2011) and dark fruit (2012) flavours, as well as bitterness, body and length (2011) and astringency (2011, 2012); the latter taste and mouthfeel-related responses were primarily confined to the T1 wines. Red fruit and herbaceous flavours decreased with delayed harvest in 2011. Insufficient volumes were available for the T2 treatments in 2012 for sensory evaluation.

\section{Interactions}

Some noteworthy interactions occurred between crop level and harvest date (Supplementary Tables 3 to 6; Fig. 5). In the 2012 Pinot gris, pear flavour was enhanced by reducing the crop in the T0 and T1 wines, but it was reduced in the T2 wines (Fig. 5A). Minimal effects of crop level occurred in the 2011 Riesling T0 wines for honey aroma, peach flavour and sweetness, but reductions in these variables occurred in $\mathrm{HC} / \mathrm{T} 1$ and in $\mathrm{HC} / \mathrm{T} 2$ (peach flavour, sweetness) relative to the $\mathrm{FC}$ wines (Fig. 5B to 5D). In the 2012 Riesling, slight reductions in peach, floral and honey aromas, vanilla flavour and acidity occurred in $\mathrm{HC} / \mathrm{T} 0$ relative to the $\mathrm{FC}$ wines, while increases in $\mathrm{HC}$ relative to $\mathrm{FC}$ occurred for the peach, floral and honey aromas, and for the vanilla flavour in the $\mathrm{T} 1$ wines, and decreases in honey aroma and vanilla

TABLE 5

Impact of crop level, harvest date and enzyme treatment on aroma compounds ( $\mu \mathrm{g} / \mathrm{L})$ in Pinot gris and Riesling musts, Niagaraon-the-Lake, ON, 2011 and 2012.

\begin{tabular}{llllll}
\hline $\begin{array}{llll}\text { Pinot gris 2011 } \\
\text { Factor }\end{array}$ & Linalool $^{\text {a }}$ & Geraniol & Nerol & $\alpha$-Terpineol & Terpinolene \\
\hline Crop level (CL) & 0.02 & 1.67 & 0.67 & 0.73 & 0.003 \\
Full & 0.02 & 0.83 & 5.61 & 0.73 & ND \\
Half & NS & NS & NS & NS & NS \\
Significance & & & & \\
Harvest date (HD) & b,c & 2.52 & $0.94 \mathrm{ab}$ & $0.73 b$ & ND \\
T0 & 0.02 & 0.97 & $0.83 \mathrm{a}$ & $0.73 b$ & ND \\
T1 & 0.02 & 0.27 & $0.15 b$ & $0.74 \mathrm{a}$ & 0.005 \\
T2 & 0.02 & NS & $\mathbf{0 . 0 5 0}$ & $\mathbf{0 . 0 1 1}$ & NS \\
Significance & NS & & & & \\
Enzyme (E) & & 0.01 & 0.04 & 0.73 & ND \\
No enzyme & 0.02 & 2.50 & 6.24 & 0.73 & 0.003 \\
Enzyme & 0.02 & $\mathbf{0 . 0 3 8}$ & $\mathbf{0 . 0 1 8}$ & NS & NS \\
Significance & NS & NS & NS & NS & NS \\
CL x HD & NS & NS & NS & NS & NS \\
CL x E & NS & NS & NS & NS & NS \\
HD x E & NS & 1.96 & 1.66 & 0.10 & 0.063 \\
Mean standard deviation & 0 & & &
\end{tabular}


TABLE 5 (CONTINUED)

\begin{tabular}{|c|c|c|c|c|c|}
\hline \multicolumn{6}{|l|}{ Riesling 2011} \\
\hline Factor & Linalool & Geraniol & Nerol & $\alpha$-Terpineol & Terpinolene \\
\hline \multicolumn{6}{|l|}{$\overline{\text { Crop level (CL) }}$} \\
\hline Full & 0.15 & 0.46 & 0.15 & 0.76 & 0.38 \\
\hline Half & 0.79 & 0.13 & 0.49 & 0.97 & 0.26 \\
\hline Significance & NS & NS & NS & NS & NS \\
\hline \multicolumn{6}{|l|}{ Harvest date (HD) } \\
\hline T0 & 0.92 & 0.66 & 0.67 & 1.06 & $0.81 \mathrm{a}$ \\
\hline $\mathrm{T} 1$ & 0.12 & 0.05 & 0.09 & 0.74 & $0.02 b$ \\
\hline $\mathrm{T} 2$ & 0.37 & 0.16 & 0.19 & 0.80 & $0.14 \mathrm{~b}$ \\
\hline Significance & NS & NS & NS & NS & $<0.0001$ \\
\hline \multicolumn{6}{|l|}{ Enzyme (E) } \\
\hline No enzyme & 0.34 & 0.13 & 0.29 & 0.84 & 0.55 \\
\hline Enzyme & 0.60 & 0.45 & 0.35 & 0.89 & 0.09 \\
\hline Significance & 0.043 & NS & 0.004 & 0.0006 & $<0.0001$ \\
\hline CL x HD & NS & NS & NS & NS & NS \\
\hline CL x E & NS & NS & NS & NS & NS \\
\hline $\mathrm{HD} \times \mathrm{E}$ & NS & NS & NS & NS & $<0.0001$ \\
\hline Mean standard deviation & 0.511 & 0.217 & 0.229 & 0.177 & 0.166 \\
\hline \multicolumn{6}{|l|}{$\underline{\text { Riesling } 2012}$} \\
\hline Factor & Linalool & Geraniol & Nerol & $\alpha$-Terpineol & Hotrienol \\
\hline \multicolumn{6}{|l|}{$\overline{\text { Crop level (CL) }}$} \\
\hline Full & 1.88 & 3.09 & 0.58 & 1.87 & 0.67 \\
\hline Half & 2.45 & 3.33 & 0.71 & 1.90 & 0.81 \\
\hline Significance & $\mathbf{0 . 0 5 0}$ & NS & NS & NS & NS \\
\hline \multicolumn{6}{|l|}{ Harvest date (HD) } \\
\hline T0 & $1.64 \mathrm{~b}$ & $2.91 \mathrm{~b}$ & $0.39 b$ & $1.65 \mathrm{~b}$ & $0.61 \mathrm{~b}$ \\
\hline $\mathrm{T} 1$ & $1.84 \mathrm{~b}$ & $2.77 \mathrm{~b}$ & $0.57 b$ & $1.78 \mathrm{~b}$ & $0.78 \mathrm{ab}$ \\
\hline $\mathrm{T} 2$ & $3.40 \mathrm{a}$ & $4.24 \mathrm{a}$ & $1.11 \mathrm{a}$ & $2.35 \mathrm{a}$ & $0.88 \mathrm{a}$ \\
\hline Significance & $<0.0001$ & 0.0002 & $<0.0001$ & $<0.0001$ & 0.010 \\
\hline \multicolumn{6}{|l|}{ Enzyme (E) } \\
\hline No enzyme & 1.86 & 1.86 & 0.60 & 1.77 & 0.74 \\
\hline Enzyme & 2.52 & 4.58 & 0.70 & 2.00 & 0.76 \\
\hline Significance & 0.013 & $<0.0001$ & NS & 0.029 & NS \\
\hline CL x HD & NS & NS & NS & 0.003 & NS \\
\hline CL x E & NS & NS & NS & NS & NS \\
\hline $\mathrm{HD} \times \mathrm{E}$ & NS & NS & NS & NS & $<0.0001$ \\
\hline Mean standard deviation & 0.905 & 0.879 & 0.239 & 0.368 & 0.286 \\
\hline
\end{tabular}

${ }^{a}$ Main effects (total terpenes) for each factor level were calculated based on the analysis of variance using crop level $\mathrm{x}$ harvest date $\mathrm{x}$ enzyme $\mathrm{x}$ replicate as a basis for the error term.

${ }^{\mathrm{b}}$ Means followed by different letters are significantly different, $\mathrm{p}<0.05$, Duncan's multiple range test.

${ }^{c}$ T0, T1, T2: Normal commercial harvest, three weeks after T0, six weeks after T0 respectively. 


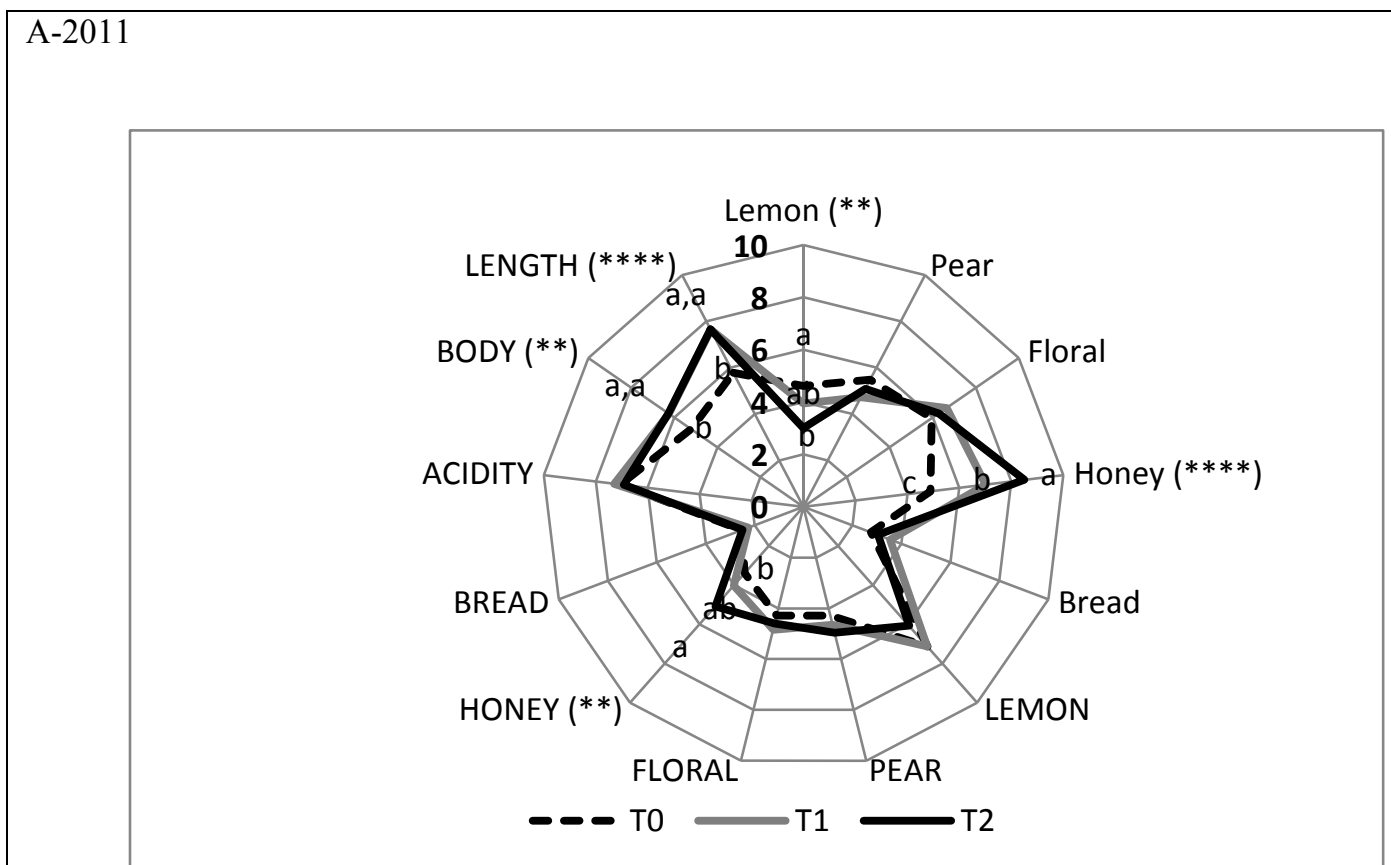

B-2012

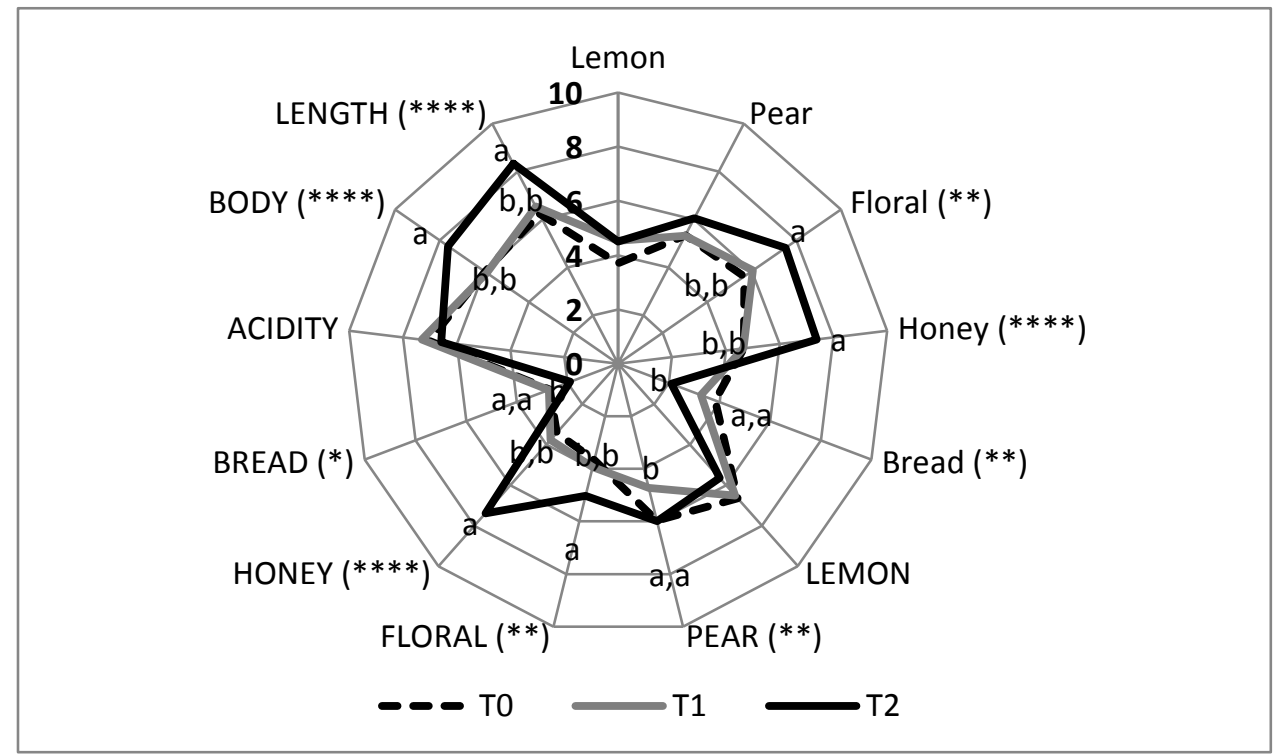

FIGURE 1

Impact of harvest date on aroma (lowercase) and flavour (uppercase) descriptors of Pinot gris wines in 2011 and 2012, Pondview Estate Winery, Virgil, ON. T0, T1, T2: Normal commercial harvest, three weeks after T0, six weeks after T0 respectively.

flavour occurred in the $\mathrm{HC} / \mathrm{T} 2$ wines relative to $\mathrm{FC}$ (Fig. 5E to 5I). In the 2011 Cabernet franc, decreases in dark fruit aroma, and red and dark fruit flavours occurred in the $\mathrm{HC} /$ $\mathrm{T} 2$ relative to the $\mathrm{FC}$ wines (Fig. $5 \mathrm{~J}$ to $5 \mathrm{~L}$ ), and the $\mathrm{HC} / \mathrm{T} 0$ wines had higher red fruit aroma $v s$ FC/T0 in 2012 (Fig. $5 \mathrm{M})$. Bell pepper aroma was noticeably reduced in the 2011 Cabernet Sauvignon HC/T0 vs the FC wines (Fig. 5N). A slight diminishment in dried fruit aroma and enhancement of red fruit flavour occurred in Cabernet Sauvignon HC/T0 $v s$ the FC counterparts (Fig. $5 \mathrm{O}$ to $5 \mathrm{P}$ ). For all of the most significant attributes, the judge $\mathrm{x}$ crop-level and judge $\mathrm{x}$ harvest date interactions were not significant, indicating that the judges were in agreement in how they rated the attributes among products (Supplementary Tables 3 to 6).

\section{Principal component analysis: Pinot gris}

The first two components (PCs) accounted for $64.6 \%$ of the variability in the data, with $\mathrm{PC} 1$ comprising $45.9 \%$ and $\mathrm{PC} 2$ $18.7 \%$ (Fig. 6A and 6B). Treatments were separated well, with all $\mathrm{T} 2$ wines located on the positive side of PC1 and 


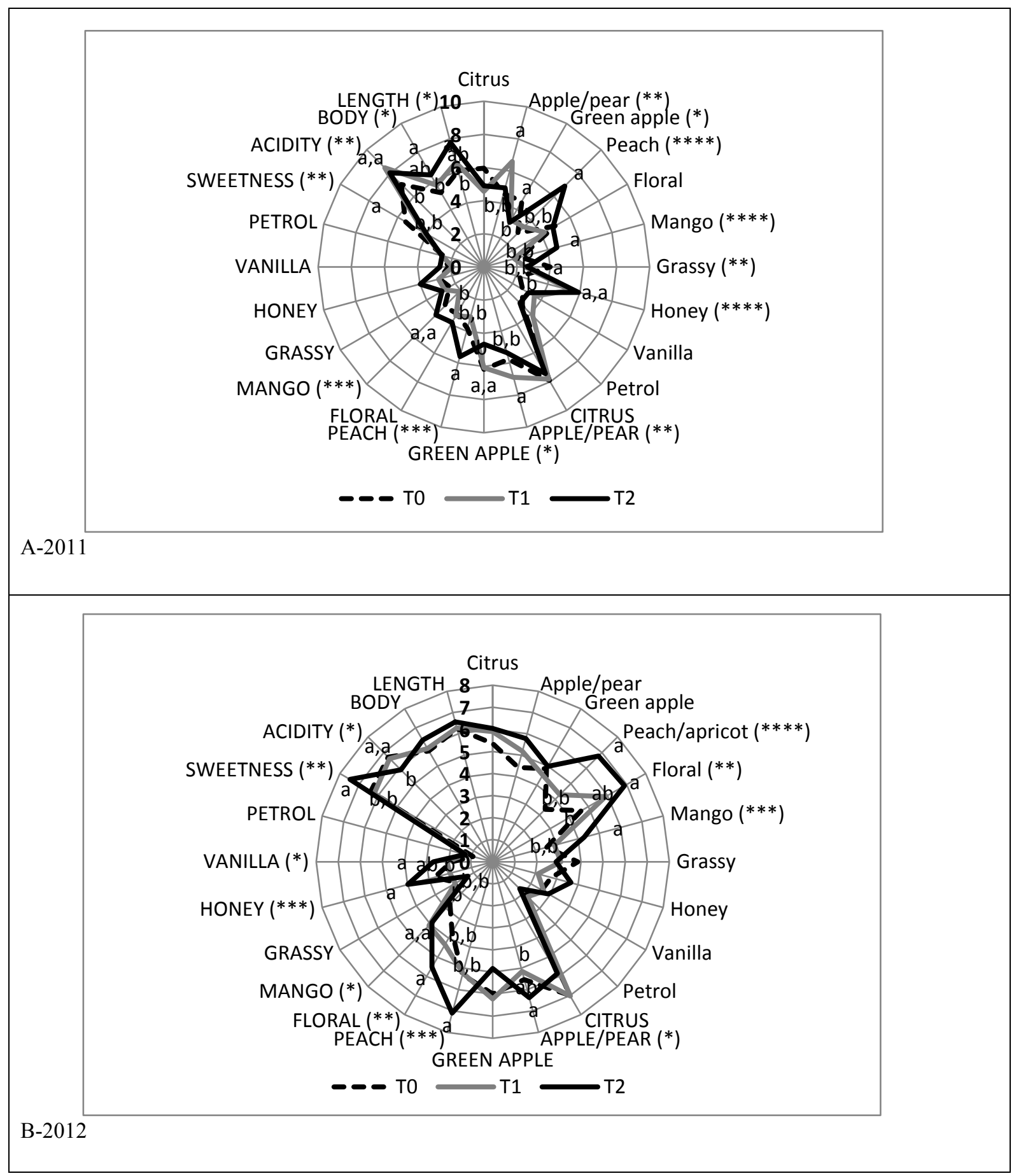

FIGURE 2

Impact of harvest date on aroma (lowercase) and flavour (uppercase) descriptors of Riesling wines in 2011 and 2012, Pondview Estate Winery, Virgil, ON. T0, T1, T2: Normal commercial harvest, three weeks after T0, six weeks after T0 respectively.

associated with pear, floral and honey aroma and flavour, as well as with body and length. All T0 and most T1 wines were positioned on the negative side of PC1 and were associated with lemon and bread aroma and flavour, as well as with acidity.

\section{Riesling}

The first two PCs accounted for $67.0 \%$ of the variability in the data, with PC1 comprising $40.0 \%$ and PC2 27.0 (Fig. 6C and 6D). Treatments were separated well, with all T0 wines located in the lower left quadrant and associated with grassy aroma and green apple flavour. Three of the four T1 wines were on the negative side of PC1 and shared some attributes with T0 wines, in addition to petrol aroma and flavour, grassy and citrus flavours, and acidity. Three of the four T2 wines plus HC/T1 (2012) were located on the positive side of PC1 and were characterised by floral, mango and peach aromas and flavours, as well as by sweetness, body and length, while two 2011 wines (one each of T1 and T2) were located adjacent to PC2 and were characterised by a honey aroma. 


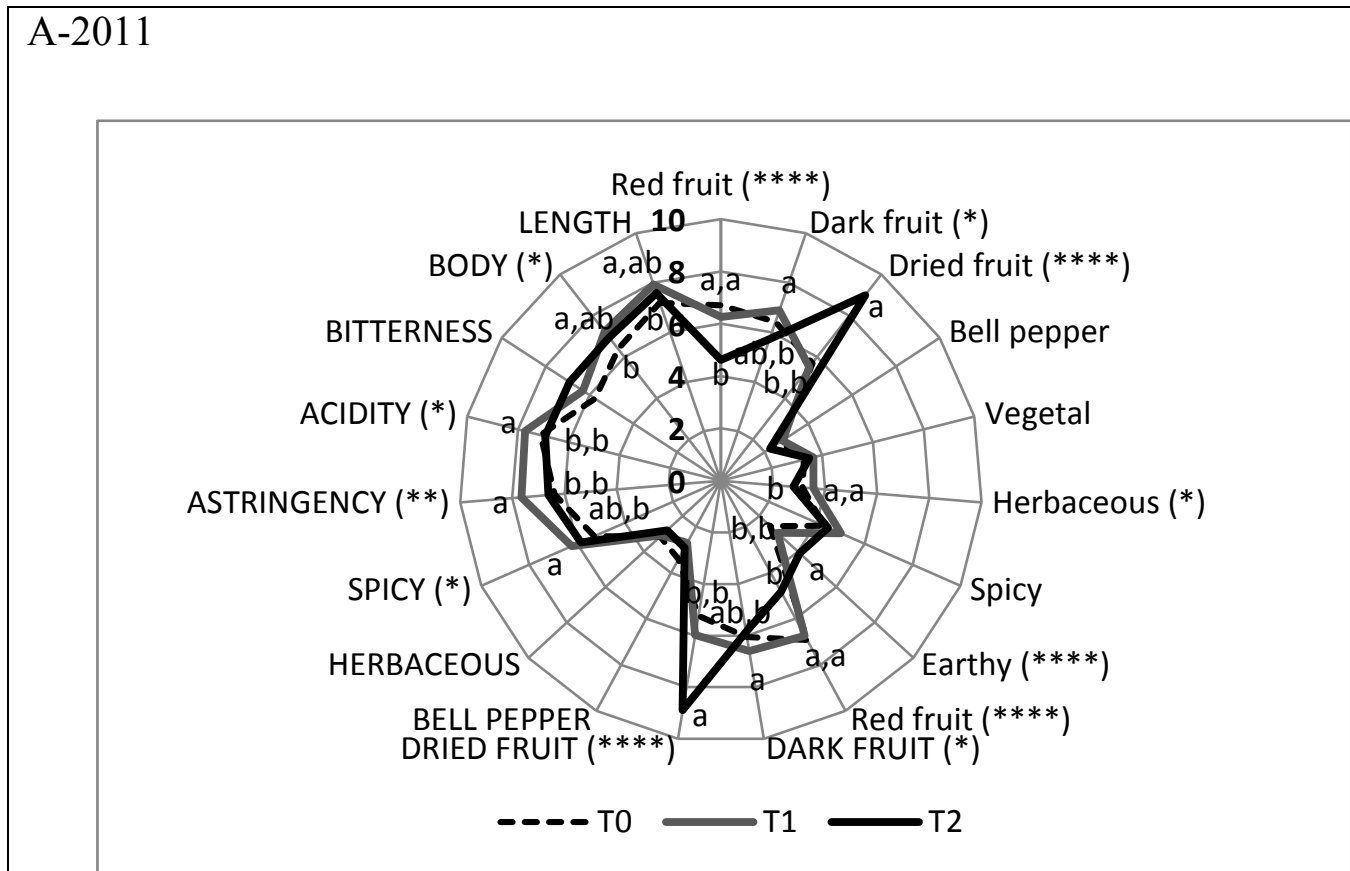

\section{B-2012}

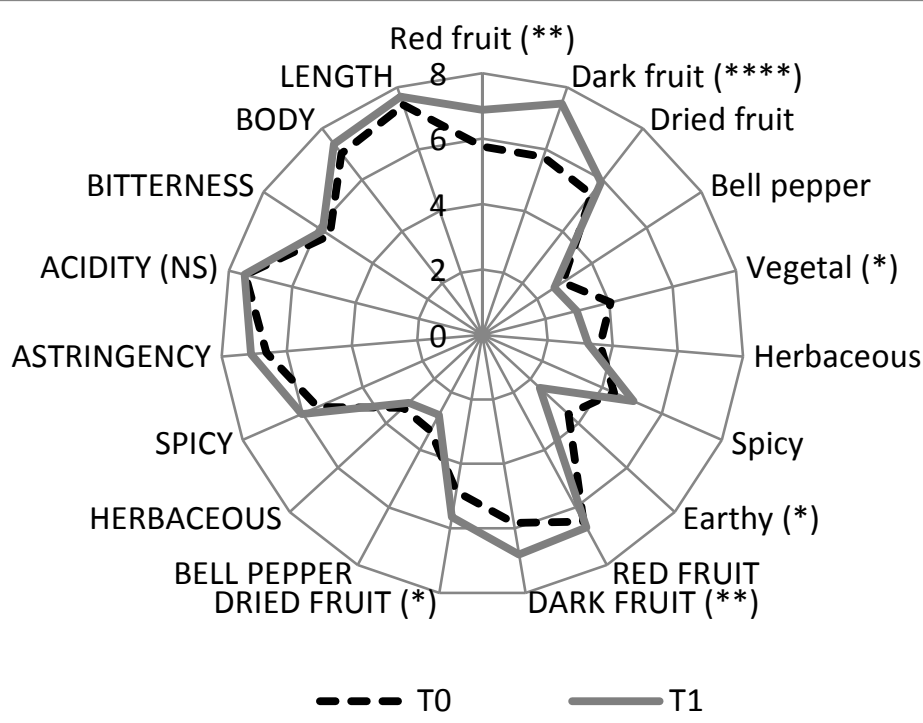

FIGURE 3

Impact of harvest date on aroma (lowercase) and flavour (uppercase) descriptors of Cabernet franc wines in 2011 and 2012 , Pondview Estate Winery, Virgil, ON. T0, T1, T2: Normal commercial harvest, three weeks after T0, six weeks after T0 respectively.

\section{Cabernet franc}

The first two PCs accounted for $67.6 \%$ of the variability in the data, with PC1 comprising 39.0\% and PC2 28.6\% (Fig. 6E and $6 \mathrm{~F}$ ). Treatments were separated well; all T0 wines were located on the negative side of PC2 and characterised by bell pepper, vegetal and herbaceous descriptors and, to a lesser extent, by red fruit. All the T1 wines were located in the upper right quadrant and associated with dark fruit and spicy descriptors, as well as length and body. The T2 wines (2011) were located in the upper left quadrant and associated with dried fruit, earthy and bitter descriptors.

\section{Cabernet Sauvignon}

The first PCs accounted for $72.8 \%$ of the variability in the data, with PC1 comprising $41.8 \%$ and PC2 $31.0 \%$ (Fig. $6 \mathrm{G}$ and $6 \mathrm{H})$. The $\mathrm{T} 0$ and $\mathrm{T} 1$ wines were grouped together on 


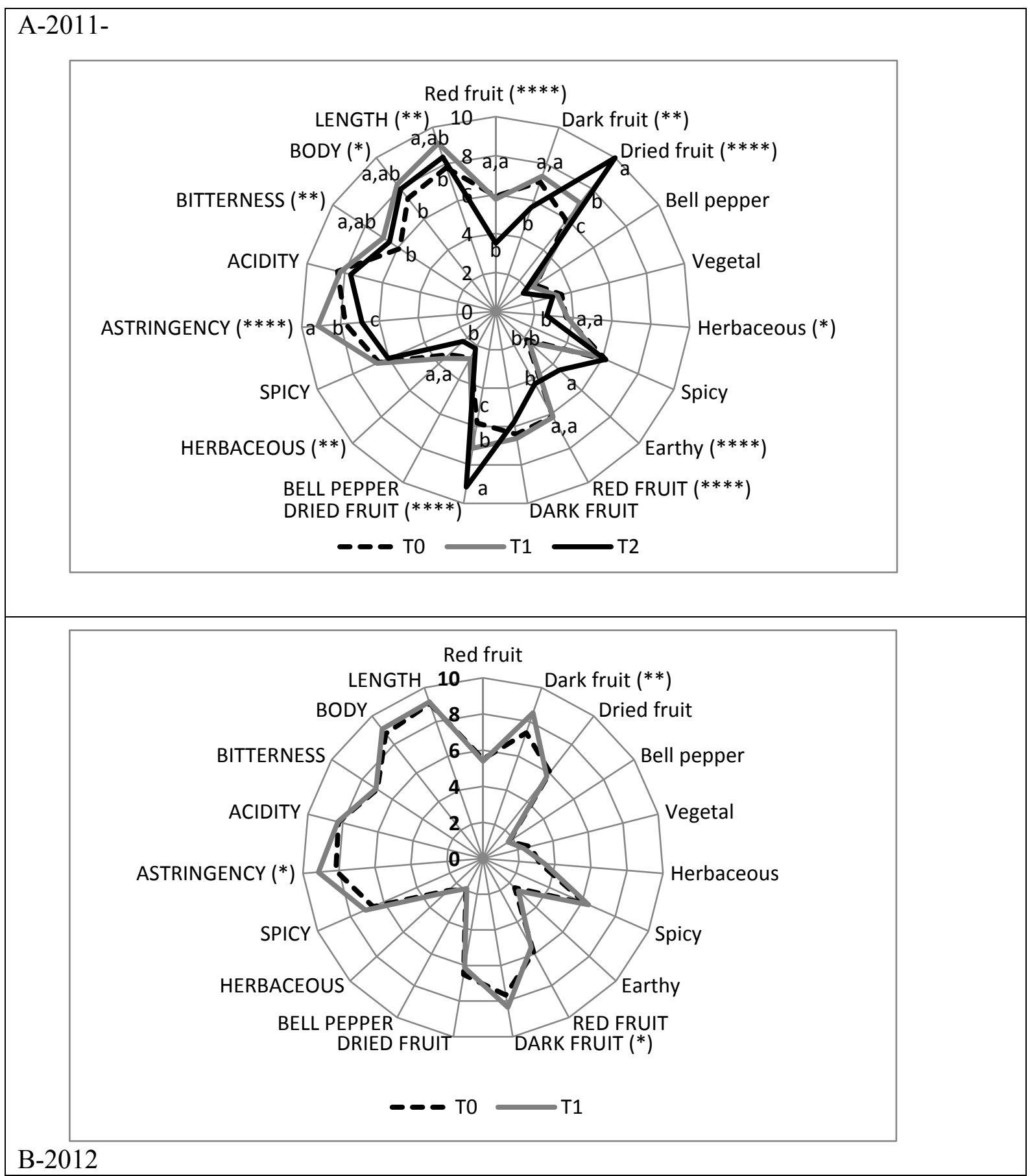

FIGURE 4

Impact of harvest date on aroma (lowercase) and flavour (uppercase) descriptors of Cabernet Sauvignon wines in 2011 and 2012, Pondview Estate Winery, Virgil, ON. T0, T1, T2: Normal commercial harvest, three weeks after T0, six weeks after T0 respectively.

the positive side of $\mathrm{PC} 1$ and split somewhat according to vintage in PC2, with 2012 on the negative side of PC2 and characterised by dark fruit, spicy, astringent, body and length, and 2011 on the positive side of PC2 and associated with bell pepper, vegetal, herbaceous, red fruit and acidity descriptors. T2 wines (2011) were on the negative side of PC1 and were associated with earthy and dried fruit descriptors.

\section{DISCUSSION}

Crop reduction led to minor changes in vine size, berry weight, and basic berry, must and wine composition (e.g. Brix, titratable acidity (TA), $\mathrm{pH}$, ethanol, anthocyanins and phenols; Moreno Luna et al., 2017). However, a delayed harvest date reduced yield, cluster weight and berry weight (due to dehydration), and increased berry and must Brix, anthocyanins and phenols (Moreno Luna et al., 2017). There was also evidence of sour rot that necessitated the sorting 

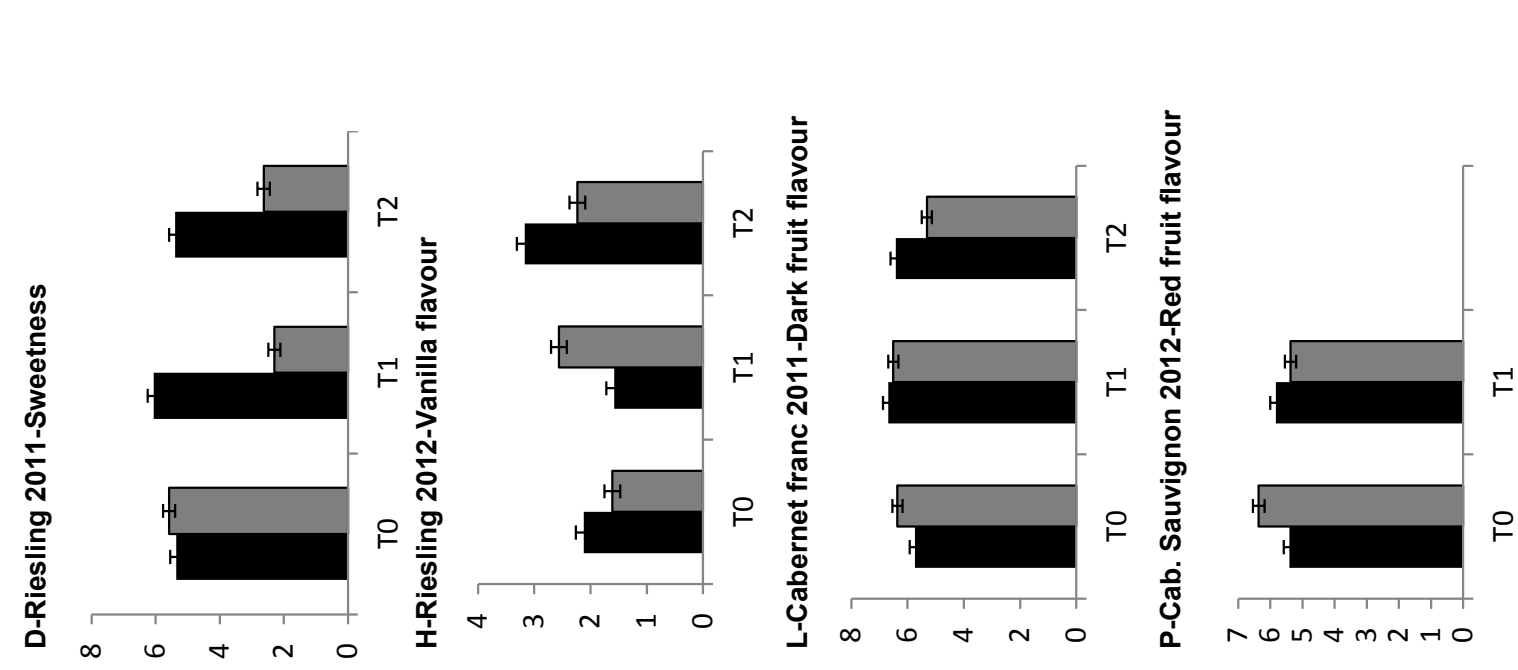

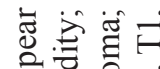

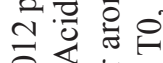

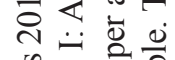

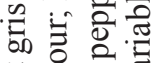

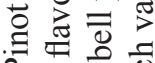

丞

< $\overline{\mathrm{Z}} \overline{\mathrm{O}}$

خั

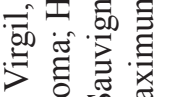

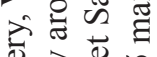

จे

3 选

节

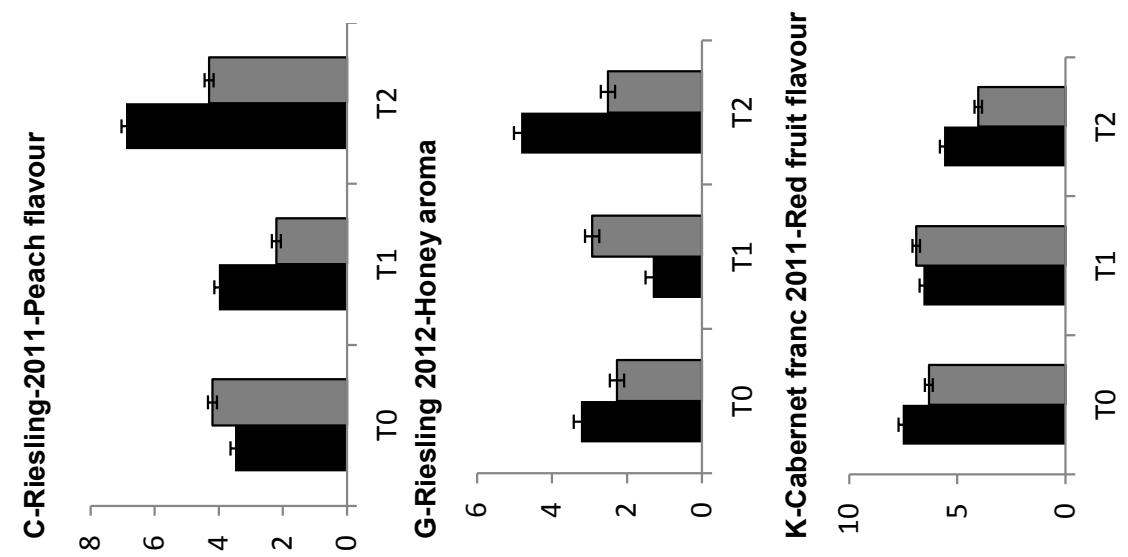

II

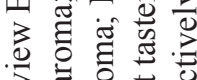

要

○

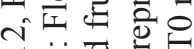

穴仙

范

官

की

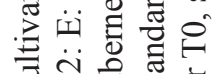

nu+mn-0 n

되 눌

.

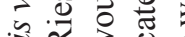

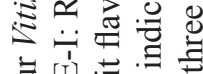
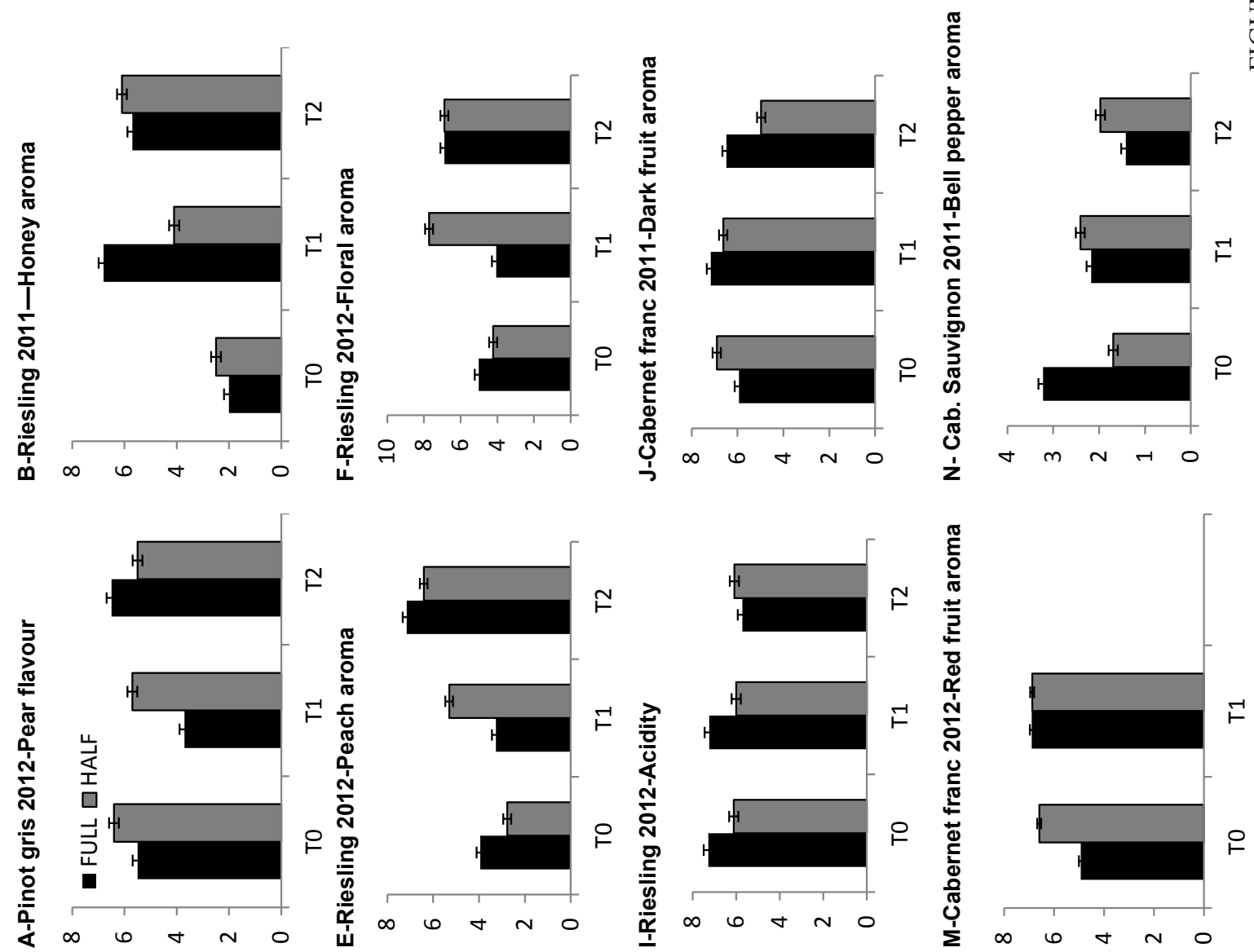

论芯芯

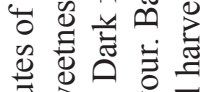

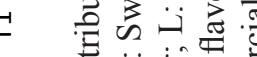

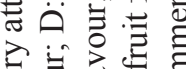

خี่

गे

至

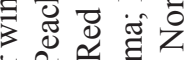

o 들

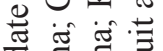

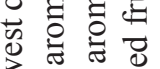

氠 层节

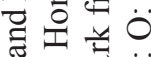

$\stackrel{\overrightarrow{0}}{\vec{D}} \ddot{\tilde{n}}$

으음

on 으. ․ㅐ

屯ี

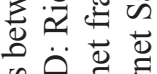

ปิ

记元

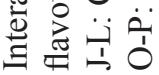




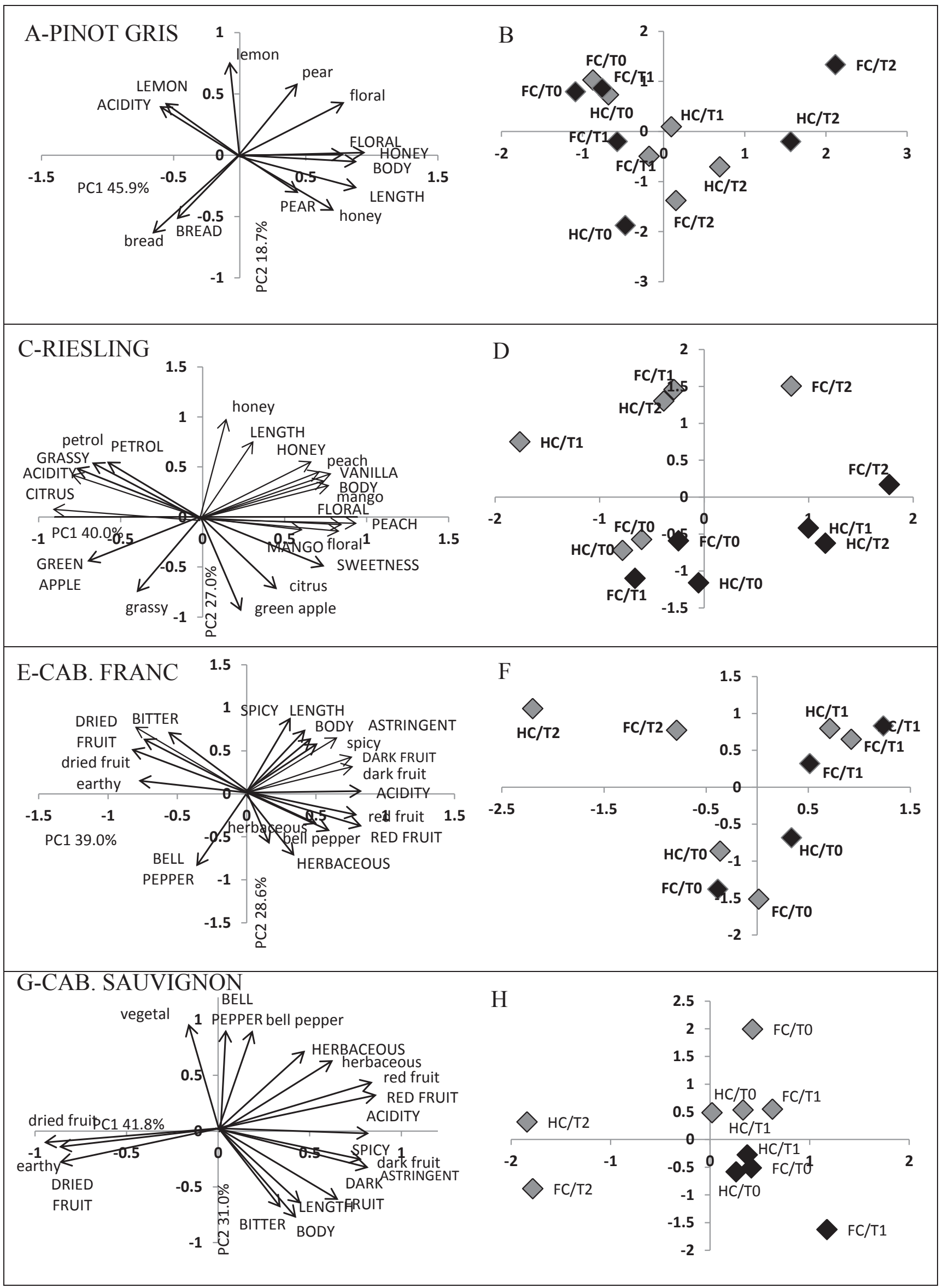

FIGURE 6

Principal components analysis of wine sensory attributes of four Vitis vinifera cultivars, 2011 and 2012, Pondview Estate Winery, Virgil, ON. A, B: Pinot gris; C, D: Riesling; E, F: Cabernet franc; G, H: Cabernet Sauvignon. Grey markers: 2011 wines; black markers: 2012 wines. 
of fruit prior to winemaking, particularly in Pinot gris and Riesling (Moreno Luna et al., 2017). An enhanced soluble solids concentration plus the effects of climatic conditions were associated with increased concentrations of wine volatile compounds. Wines made from dehydrated grapes normally contain more terpenes and norisoprenoids than the non-dehydrated controls (Moreno et al., 2008), while crop reduction can increase free and bound terpenes (Reynolds et al., 2007).

\section{Wine aroma compounds \\ Monoterpenes}

Terpenes such as linalool, geraniol, $\alpha$-terpineol and citronellol were present in all cultivars, with some exceptions. Only citronellol and terpinolene were found in Pinot gris, Citronellol was only present at T2 in 2011 and not detected in 2012, while terpinolene decreased with delayed harvest but was only present in $\mathrm{HC}$ in 2011, and at both crop levels at T2 in 2012. The effect of dehydration likely played a role in the accumulation and possibly the biosynthesis of this compound (Moreno et al., 2008). Citronellol decreased in Cabernet franc with harvest date, until non-detectable at T2; this reduction in concentration could be explained by possible changes in grape berry metabolism when desiccation occurs (Kays, 1997). In Cabernet Sauvignon, citronellol was only detected in 2012 and only in HC in Cabernet franc in 2011. Citronellol and other terpenes are normally odour-active (Mateo \& Jiménez, 2000), and their OAVs were $>1$ in wines from delayed harvest fruit.

Linalool, geraniol and $\alpha$-terpineol were detected in Riesling, with the last two being present only in 2011. The linalool concentration increased with harvest date in 2011, but was only present in the T2 wines in 2012. This increase with delayed harvest may be a reflection of dehydration occurring in the grapes (Sponholz, 1993), since reductions in berry weight and yield occurred at T2. Linalool concentrations were higher than reported in the literature (4.7 to $307 \mu \mathrm{g} / \mathrm{L}$ for young white wines; Guth, 1997; Escudero et al., 2004). No geraniol was detected in Riesling in 2012, but in 2011 a decline was noted with delayed harvest, with values consistent with those in the literature $(26 \mu \mathrm{g} / \mathrm{L}$ in juice; Gunata et al., 1985). $\alpha$-Terpineol responded similarly to geraniol; it was detected in Riesling in 2011 only, with increases due to delayed harvest. Noble rot following infection by $B$. cinerea generates the conversion of linalool, geraniol and nerol to less-volatile compounds such as $\alpha$-terpineol (Bakker \& Clarke, 2012). This could explain the increase in 2011, which was a wetter year during harvest compared to the dry and cold 2012 season (Moreno Luna et al., 2017), and thus the grapes would have been less susceptible to infection.

Other terpenes had $\mathrm{OAVs}>1$ in most cases, making them odour-active where they were present. Terpinolene (plastic/ pine) was detected in the $\mathrm{HC}$ wines at all harvest dates for Pinot gris in 2011, but only at T2 in 2012. Terpinolene is generated by a transformation due to an acid-catalysed rearrangement of nerol and linalool (Marais, 1983). Nerol and linalool are more sensitive to acid conditions and temperature (Marais, 1983), producing less aromatic compounds such as terpinolene and limonene. In both years, the increase in TA resulting from the concentration of organic acids associated with delayed harvest (Moreno Luna et al., 2017) could have elicited the formation of this component. In Riesling, this compound was mainly present during 2011, a warmer, wet year, with an increase in the HC treatments and an increase with delayed harvest. In 2012 it was only detected in the T2 treatments, at concentrations closer to those in 2011.

\section{Norisoprenoids}

The majority of grape norisoprenoids have 13 carbons and are derived from the enzymatic cleavage of carotenoids (Dunlevy et al., 2009). The generation of norisoprenoids from carotenoid breakdown occurs via chemical, photochemical and oxidase-coupled degradation. Carotenoids decline under high fruit exposure and enhanced fruit temperatures; e.g. carotenoids declined in concentration under thermo-tunneldried treatments (Chkaiban et al., 2007). $\beta$-Damascenone was detected in all cultivars in all treatments, and a reduction occurred in all cultivars with delayed harvest. The effect of stress due to the desiccation of grapes that are hung for prolonged periods, particularly at $\mathrm{T} 2$, could possibly be responsible for the diminished enzyme capacity to generate breakdown products. In general, OAVs for $\beta$-damascenone were $>1$; it was once considered exclusively a component that adds nuances to different cultivars, but its role is now recognised to be one of enhancement of the odour activity of other compounds (Escudero et al., 2004).

\section{Esters}

The four most abundant esters were ethyl acetate, isoamyl acetate, ethyl caproate and ethyl caprylate. The majority were affected more by delaying the harvest date than reducing the crop. While ethyl acetate and ethyl butyrate increased with delayed harvest in almost all cases, ethyl caproate and hexyl acetate were reduced. They are formed primarily during fermentation, although they could be present in small amounts in grapes (Dunlevy et al., 2009). Differences in Brix prior to fermentation, particularly at $\mathrm{T} 2$ and subsequent to fermentation, may have led to their generation. These increases may have been linked specifically to the Brix increase resulting from dehydration (Bellincontro et al., 2004; Costantini et al., 2006), and subsequent fermentation and production of ethanol and higher alcohols, which elicit the synthesis of acetates of higher alcohols (Zamora, 2009). Changes in esters could also be generated by a reestablishment of chemical equilibrium relative to the percentage of ethanol and acids in the wines immediately after fermentation (Bakker \& Clarke, 2012), coupled with changes in berry metabolism when water loss occurred (Zamboni et al., 2008).

Two different groups of esters were detected in the wines, namely acetates of ethanol and higher alcohols, e.g. ethyl acetate, isoamyl acetate and hexyl acetate, and esters of fatty acid metabolites and ethanol, e.g. ethyl butyrate, caproate, caprylate, heptanoate and decanoate. Acetate esters are synthesised from acetyl-CoA and higher alcohols (Zamora, 2009), and increased Brix associated with delayed harvest, followed by the enhanced production of ethanol and higher alcohols, could be responsible for the changes in concentration. Increased ethyl acetate (pineapple, nail polish 
remover) in almost all wines is consistent with the findings of Zamboni et al. (2008), who linked this phenomenon to a metabolic stress response when $11.7 \%$ of water was lost from the grapes. However, a decrease in Riesling was detected in 2012 in relation to extended harvest date, but only in the T2 wines; T2 berry weight was much lower compared to that of the other harvest dates (Moreno Luna et al., 2017). The T2 Riesling wines in 2011 had the highest concentrations of ethyl acetate, although they were not noticeably faulted. Isoamyl acetate (banana) was affected by both factors; in white cultivars, its concentration was reduced with delayed harvest, consistent with the reduction in isoamyl alcohol in the same cultivars, while crop reduction led to increases in the 2012 Cabernet franc. Isoamyl alcohol (whisky/malt) did not change in relation to crop reduction or harvest date in Cabernet Sauvignon; however, an increase in acetates of carboxylic acids (e.g. ethyl acetate), linked to an increase in volatile acidity, was most likely associated with the extremely late harvest that extended into early December. Vintage had an apparent impact in white cultivars, with a marked increase in concentration in many esters in 2012, linked to increased Brix in the grapes and ethanol in the wines; red cultivars remained unchanged. Hexyl acetate (herbal, fruity) was present only in white cultivars and declined with delayed harvest. Concentrations were as high as $6100 \mu \mathrm{g} / \mathrm{L}$ in Pinot gris and 2600 to $5700 \mu \mathrm{g} / \mathrm{L}$ in Riesling, which are consistent with the findings of Rapp and Mandery (1986), and higher concentrations represented OAVs $>1$.

The second group of esters, consisting primarily of ethyl esters, are formed via the lipoxygenase (LOX) pathway and derived from the breakdown of fatty acids, leading to the production of several alcohols, aldehydes and carboxylic acids (Schwab et al., 2008; Zamora, 2009). Effects on the enzymatic pathways associated with the formation during fruit maturity and vinification, and the associated ethanol production, could explain treatment differences in this trial. During vinification, grape aliphatic compounds are depleted and converted to alcohols and esters, usually with positive sensory attributes (Dunlevy et al., 2009). The LOX pathway involves several enzymes that oxidise and cleave polyunsaturated fatty acids to form aldehydes, which are subsequently reduced to alcohols and esterified in the presence of short-chain carboxylic acids such as acetic acid (Schwab et al., 2008; Dunlevy et al., 2009). Enzyme activities in the LOX pathway (e.g. LOX, hydroperoxide lyase, alcohol dehydrogenase (ADH)) are active in maturing fruit and during fermentation, when they come into contact with fatty acids in the presence of oxygen (Schwab et al., 2008; Chkaiban et al., 2007).

Ethyl butyrate (apple) was always found with OAVs $<1$, suggesting that it was not odour-active. Ethyl caproate (apple peel) was found in higher concentrations in white cultivars, consistent with the findings of Bakker and Clarke (2012); i.e. 60 to $2000 \mu \mathrm{g} / \mathrm{L}$ in white wines and 60 to $130 \mu \mathrm{g} / \mathrm{L}$ in red wines. Although it was reduced in almost all wines with delayed harvest date, this could be linked to a reduction in fatty acids in the grapes prior to fermentation, particularly at T2. A vintage effect was also apparent. Higher concentrations were detected in Pinot gris and Riesling in 2012 vs 2011, and reductions were observed in 2012 vs 2011 for Cabernet franc and Cabernet Sauvignon. The reduction in red wines could also have been linked to a hydrolysis of this ester due to malolactic fermentation, or to the action of indigenous Lactobacillus bacteria on the fruit (Costantini et al., 2009). The $\mathrm{pH}$ values of these wines were $>3.4$, providing a feasible environment for their growth (Costantini et al., 2009), and $\mathrm{pH}$ increased with delayed harvest, while ethyl caproate decreased. Ethyl caprylate (fruit, fat) was found in higher concentrations in white wines relative to the findings in the literature (1 100 to $5100 \mu \mathrm{g} / \mathrm{L}$; Bakker \& Clarke, 2012), but was lower in red wines (1 000 to $6000 \mu \mathrm{g} / \mathrm{L}$; Bakker $\&$ Clarke, 2012), and this suggests higher odour activity of ethyl caprylate in white cultivars, particularly Riesling, since it was not detected in Pinot gris in 2011. Cabernet franc had increased ethyl caprylate with delayed harvest date, but Cabernet Sauvignon was not affected; again, vintage led to differences, with higher concentrations in the red cultivars in 2011 vs 2012. Ethyl heptanoate (fruity) was mostly detected in red cultivars, with increases as a result of delayed harvest; however, all OAVs were $<1$, suggesting it was not odour-active. The same behaviour was observed for diethyl succinate (wine, fruit); even though it was affected more in red cultivars than in white ones, in both cases it had a OAV $>1$. Ethyl decanoate (grape) was not odour-active for red cultivars, but was slightly $>1$ for white cultivars, with an increase with delayed harvest date in 2012. Ethyl phenylacetate (fruit, sweet) had OAVs $>100$ for Pinot gris and Riesling, and this increased relative to harvest date for Pinot gris in both seasons.

\section{Higher alcohols}

The production of higher alcohols is linked to the production of ethanol in wines. They can be derived from amino acids (Zamora, 2009). Some ADH enzymes in fruit and yeasts are responsible for catalysing the reduction of aliphatic aldehydes to alcohols (Dunlevy et al., 2009). In grapes, stress conditions have been associated with an increase in ADH activity (Dunlevy et al., 2009). ADH in grape berries can be activated at certain levels of water loss (Costantini et al., 2006). This could explain the higher concentrations of compounds such as isobutyl alcohol (wine solvent, bitter); this component was reduced in the 2011 Pinot gris with delayed harvest, which could potentially have reduced pungency and bitterness (Bakker \& Clarke, 2012). Isobutyl alcohol was not detected in the 2012 Pinot gris. Higher concentrations were found in the 2011 Riesling in comparison with what was observed in the literature (6 000 to 174000 $\mu \mathrm{g} / \mathrm{L}$; Bakker \& Clarke, 2012), and was $~ 300000 \mu \mathrm{g} / \mathrm{L}$ in 2012; in both years, it was present only in the T2 wines, which could be related to the presence of sour rot, which elicits the formation of this compound in Riesling grapes (Zoecklein et al., 2010). Similar concentrations were found in red cultivars in both years, without any trend with respect to viticultural treatments; it was only lacking in the 2011 Cabernet Sauvignon. Phenylethanol (honey, spice, rose/lilac) was detected in all wines and did not display a general trend between cultivars; it was found at concentrations consistent with those in the literature (9 000 to $153000 \mu \mathrm{g} / \mathrm{L}$ in red wines, 13900 to $86800 \mu \mathrm{g} / \mathrm{L}$ in white wines; Escudero et al., 2004). OAVs for white cultivars were slightly $>1$, but higher 
for red cultivars, which suggests that they had a greater impact in red cultivars. Isoamyl alcohol was present in red cultivars in 2011, with concentrations higher than those in the literature, and with OAVs $>40$. The generation of this compound may have occurred due to sour rot and/or Botrytis infection as a result of an extended harvest date; in Cabernet franc, however, delayed harvest resulted in its reduction. Hexanol (leafy, grassy) was present in all cultivars; in white wines, concentrations were barely enough for odour activity, but in red wines concentrations were higher than reported in the literature, from 2100 to $13200 \mu \mathrm{g} / \mathrm{L}$, which might have generated an increase in the herbaceous aroma (Guth, 1997). In these red wines, decreases in hexanol due to delayed harvest probably affected the flavour of the wines.

Other higher alcohols were found sporadically in some wines, and their presence could be related to microbial growth on grapes as a result of an extended harvest date, which could have enhanced the production of polyols during berry shrinking and associated water loss (Sponholz, 1993). There was a production of high and odour-active amounts of nonanol (fat, green) in Cabernet Sauvignon wines, and increased with harvest date in 2011, but was only detected in 2012 in the HC/T2 samples. This was related to climatic conditions during harvest; 2011 was a wet and warm year, while the hot and dry 2012 season could explain the formation of this alcohol. Heptanol (chemical, green) was also present in a few T2 samples in 2012 Riesling; this compound was also detected in 2012 Cabernet franc, with OAVs $>1$. Similar trends were observed in relation to 1-octanol in the 2012 Pinot gris, with OAVs $>1$, making them odour-active.

\section{Volatile acids}

The trend with volatile acids was the same in all cultivars, with a reduction in concentration with delayed harvest; this reduction probably affected the wine aroma, since all compounds from this group have an odour quality ranging between sweat, cheese and rancid fat. Hexanoic and decanoic acids had OAVs $<1$, which suggests they were not odouractive. Crop reduction can increase the concentration of malic acid (Bravdo et al., 1984); however, in this experiment, some volatile acids (e.g. hexanoic, octanoic and decanoic acids) were reduced primarily by extended harvest date, which could be linked to a possible decrease in TA and a change in the acidic composition of the grapes, consistent with the findings of Casassa et al. (2013), who reported diminished TA and malic acid, and increased acetic and lactic acids associated with extended harvest date.

\section{Other compounds}

Benzaldehyde (almond, burnt sugar) is often associated with defective wines (Bakker \& Clarke, 2012). It is probably formed by the oxidation of benzyl alcohol, or by the action of microorganisms on aromatic amino acids (phenylalanine), phenol compounds or secondary metabolites, such as phenyl acetic acid and $p$-hydroxybenzoic acid (Dunlevy et al., 2009). Benzaldehyde was detected at T2 in a few wines, such as the 2012 FC/T2 Pinot gris, which had an OAV of 27, while all the other wines had OAV values considerably lower. Diethyl acetal (fruit, cream) followed a similar trend in relation to benzaldehyde, with a high presence in T2 and with OAVs sufficiently high that they likely had a considerable impact on the wine sensory profile.

\section{Must terpenes}

The knowledge that monoterpenes and other compounds existed as both aglycones and glycosides was first mentioned by Cordonnier and Bayonove (1974), and has since been confirmed widely (Gunata et al., 1985). The percentage of terpenes present as glycosides is normally considerably higher than that of aglycones, but this varies substantially between skin, juice and pulp, with the greatest proportions found in the skins (Marais, 1983). Moreover, the proportion of glycosides accountable for individual terpenes varies widely; with free $v s$ bound terpenes in Riesling juice reported by Gunata $e t$ al. (1985) as $58 v s 276 \mu \mathrm{g} / \mathrm{L}$, with values for individual terpenes as follows: linalool (19 vs 87); geraniol (26 vs 65); nerol (5.4 vs 10.3); $\alpha$-terpineol (7.4 vs 114). Individual values reported in Riesling juice by Marais and Van Wyck (1986) were all $<10 \mu \mathrm{g} / \mathrm{L}$, whereas those of Michlmayr et al. (2012) were (free $v s$ total, $\mu \mathrm{g} / \mathrm{L})$ : linalool (0.96 vs 20); geraniol (0.35 vs 17); nerol (0.5 vs 20); $\alpha$-terpineol (7.4 vs 114). Ranges in this trial were therefore lower than those found by Gunata et al. (1985), but consistent with those of the other aforementioned studies, and suggested high concentrations of bound linalool, geraniol, nerol and $\alpha$-terpineol. Both free and bound terpenes are responsive to viticultural practices, including crop size (Reynolds \& Wardle, 1989; Reynolds et al., 2007) and harvest date (Marais, 1987). Data from this trial suggest that cluster thinning had little impact on must terpenes in Riesling and Pinot gris, but delayed harvest substantially increased most terpenes.

\section{Sensory analysis \\ Crop level}

Cluster thinning had very few effects on wine sensory attributes across the four cultivars and two seasons. Many studies have been done that confirm the beneficial effects of reducing crop level on wine quality in cool regions (Reynolds et al., 1994, 2007; Preszler et al., 2013). However, the literature suggests that the effects of cluster thinning on wine sensory properties are somewhat ambiguous. Cluster thinning enhanced ripe fruit and reduced green fruit character in Riesling (Reynolds et al., 1994), and "low crop" wines could be identified by a sensory panel using a sorting process (Preszler et al., 2013), but cluster thinning led to only small increases in concentrations of odour-active constituents in Riesling wines in NY state (Meyers et al., 2013). Likewise, thinning Riesling and Chenin blanc vines in Washington state led to few positive changes in berry composition that could have had an impact on wine quality (Keller et al., 2005). Cluster thinning increased lychee and dried fruit intensities and reduced acidity and vegetal attributes in Chardonnay Musqué wines (Reynolds et al., 2007), but these effects were dependent on vintage. No change in wine quality occurred in cluster-thinned Gewürztraminer (Reynolds \& Wardle, 1989), perhaps due to a paucity of corresponding differences in fundamental metrics, e.g. yield, fruit exposure and berry composition (monoterpenes excepted), between thinned and non-thinned treatments. Yield per vine had no effect on Sauvignon blanc sensory quality (Naor et al., 
2002), but wine scores decreased with increasing crop load (Ravaz Index; ratio of yield per vine to weight of cane prunings). This demonstrates that lowering crop size is of no consequence, unless it contributes positively to the balance between vine size and crop size. It is also clear from this and previous studies that white wine cultivars are not particularly sensitive to changes in crop size. Crop loads (full crop, half crop) in this study were 6.5 and 4.7 (2011) and 13.5 and 10.1 (2012) respectively for Pinot gris, and 8.0 and 4.9 (2011) and 11.7 and 7.6 (2012) respectively for Riesling (Moreno Luna et al., 2017); these are considered optimum (10 to 12) or undercropped $(<10)$ based on previous criteria (Bravdo et al., 1984; Kliewer \& Dokoozlian, 2005). None of these changes to crop load led to substantial changes in wine sensory quality.

Most sensory studies devoted to cluster-thinning effects have involved red wine cultivars (Bravdo et al., 1984, 1985; Reynolds et al., 1996; Chapman et al., 2004; Di Profio et al., 2011). However, as with white wine cultivars, the beneficial impact of lowering yield per vine is dependent upon a multitude of factors, including cluster exposure, berry size and degree of water deficit (Matthews \& Nuzzo, 2007). Cluster thinning Cabernet Sauvignon "slightly increased" wine quality in two of three seasons, but vineyard location played a much greater role (Ough \& Nagaoka, 1984). Three cluster-thinning levels (combined with four irrigation levels) in Cabernet Sauvignon had little impact on wine sensory variables over a five-year period; irrigation treatments had a much greater effect, and cluster thinning reduced crop loads to values $<4$ in some seasons, whereas crop loads of fullycropped treatments did not exceed 8 (Bravdo et al., 1985). Cabernet Sauvignon pruned to low node numbers produced wines with high vegetal and bell pepper and low red/black fruits, and cluster thinning had very little effect (Chapman et al., 2004). These responses were attributed to increased shade in low-crop treatments based on low node numbers, and increased berry size in cluster-thinned treatments. The metrics of potential wine quality were likewise unaffected in cluster-thinned Cabernet Sauvignon in Washington state, perhaps due to crop loads that ranged from 2.6 to 7.3 in one study (Keller et al., 2005), and from 2.9 to 5.5 in another (Keller et al., 2008). Cluster thinning Cabernet Sauvignon, Merlot, and Cabernet franc led to numerous positive sensory effects, as well as associated increases in several individual anthocyanins and phenols (Di Profio et al., 2011), but many cluster-thinning effects were enhanced when fruit-zone leaf removal was used. Reducing crop level of Cabernet Sauvignon to less than half that of the control treatments increased concentrations of isobutylmethoxypyrazine, presumably due to increases in berry size in the clusterthinned treatments (Dunlevy et al., 2013).

Other cultivars have demonstrated greater response to cluster thinning in terms of secondary metabolites and sensory attributes. Carignane vines were very responsive to cluster thinning, most likely because crop loads were reduced from a five-year mean of 19.3 in unthinned vines (range 16 to 27 ) to values ranging from 12 to 15 (moderate thinning) and 8 to 12 (severe thinning) (Bravdo et al., 1984). Merlot malvidin-3-glucoside and total phenols were increased in cluster-thinned vines, despite very low crop loads $(<3$; King et al., 2012). Tempranillo responded to crop reduction with higher wine scores following both manual cluster thinning (Gamero et al., 2014) and mechanical thinning (Diago et al., 2010), but Grenache was largely unresponsive (Diago et al., 2010). In Pinot noir, widely considered highly responsive to crop reduction, reducing the crop level in trials in British Columbia and Oregon increased colour, currant aroma, astringency and intensity of finish, independent of shoot density level and Scott Henry training (Reynolds et al., 1996). As with white wine cultivars, cluster thinning may be inconsequential sensorially, unless crop reduction leads to substantial changes in crop load and vine balance. Crop loads in this trial were 4.4 and 3.1 (2011) and 7.8 and 5.5 (2012) for Cabernet franc, and 5.0 and 4.0 (2011) and 9.3 and 5.9 (2012) for Cabernet Sauvignon (Moreno Luna et al., 2017). These crop loads were below the optimal values established by Bravdo et al. (1984) and Kliewer and Dokoozlian (2005), and this may explain a general lack of response to cluster thinning.

\section{Harvest date}

Extension of the harvest date produced numerous changes that could be considered as positive. In Riesling and Pinot gris, later harvests led to increased intensities of pear, floral and honey attributes in Pinot gris, and floral, vanilla, peach and mango attributes in Riesling. The Cabernet franc and Cabernet Sauvignon T1 and T2 wines were best described by dried fruit and earthy attributes, and also had higher astringency than the T0 wines. Delays in harvest are well known to lead to increases in monoterpenes and norisoprenoids in white wine cultivars such as Riesling (Boss et al., 2014). In red wine cultivars, attention has been directed towards both aroma compound development (Sun et al., 2011) and changes in anthocyanin and phenolic compounds (Casassa et al., 2013). In most circumstances, later harvests have resulted in reduced intensities of vegetal attributes and concomitant increases in fruity notes (Casassa et al., 2013), along with corresponding changes in astringency and bitterness (Cadot et al., 2012). In Merlot, delayed-harvest wines were described by viscosity, sweet taste and fruitderived aromas, while early-harvest wines were described by vegetal character, acidity and low colour intensity (Casassa et al., 2013). Late-harvest Maréchal Foch wines were described as fruitier than their earlier-harvested counterparts (Sun et al., 2011). In Cabernet franc, astringency, bitterness, colour intensity and alcohol increased with delayed harvest (Cadot et al., 2012).

\section{CONCLUSIONS}

The viticultural treatments imposed by this study had effects on wine aroma composition. The effects of delayed harvest on aroma compounds were substantially greater than those of crop reduction. Aroma compounds such as terpenes that are normally associated with varietal aroma in the white wine cultivars increased in concentration with delays in harvest date, making the wines more varietal-like and intense. A decrease in $\beta$-damascenone was linked with a delay in harvest date. Vintage affected numerous aroma compounds, with higher concentrations in some cases in 2012 than in 2011, perhaps linked to the higher Brix values 
in all cultivars in 2012. Riesling was less affected by vintage, since higher concentrations of terpenes were detected in 2011 than in 2012. Increases in ester concentrations were linked to delayed harvest more than crop reduction. In some cases, concentrations declined with delayed harvest, e.g. ethyl caproate, isoamyl acetate and hexyl acetate, while increases occurred for other compounds. Volatile acids were reduced with delayed harvest. Higher alcohols sometimes were affected by delayed harvest, with reductions in phenylethanol and hexanol. For hexanol, its reduction in red wines would have decreased the herbaceous odour that it characterises. However, the presence of other compounds suggested the activity of microorganisms, particularly following six weeks of extended harvest (T2 treatments). Higher concentrations of isobutyl alcohol in Riesling, and nonanol in Cabernet Sauvignon, were related to delayed harvest, particularly at T2, and might have been associated with desiccation. Overall, delayed harvest had a greater impact on most wine aroma compounds and wine sensory attributes compared to crop reduction. This is linked not only with higher concentration of sugars, but also changes in other metabolites that either increased or decreased with delayed harvest. Climatic conditions and cultivar must also be considered when extension of harvest is chosen. Despite enhancements in wine quality, treatments involving lengthy extensions of harvest date may not be economically sustainable in cool, humid climates due to crop loss from sour rot, Botrytis and dehydration.

\section{LITERATURE CITED}

Bakker, J. \& Clarke, R.J., 2012 (2nd ed.). Wine flavour chemistry. WileyBlackwell, Oxford.

Bellincontro, A., De Santis, D., Botondi, R., Villa, I. \& Mencarelli, F., 2004. Different postharvest dehydration rates affect quality characteristics and volatile compounds of Malvasia, Trebbiano and Sangiovese grapes for wine production. J. Sci. Food Agr. 84, 1791-1800

Black, J.A.K., Di Profio, F.A., Le Dauphin, V., Moreno, L.H. \& Reynolds, A.G., 2016. Impact of crop level and harvest date on anthocyanins and phenolics of red wines from Ontario. Can. J. Plant Sci. 96(6), 1045-1059.

Boss, P.K., Böttcher, C. \& Davies, C., 2014. Various influences of harvest date and fruit sugar content on different wine flavor and aroma compounds. Am. J. Enol. Vitic. 65, 341-353.

Bowen, A.J. \& Reynolds, A.G., 2012. Odor potency of aroma compounds in Riesling and Vidal blanc table wines and icewines by gas chromatographyolfactometry-mass spectrometry. J. Agric. Food Chem. 60, 2874-2883.

Bravdo, B., Hepner, Y., Loinger, C., Cohen, S. \& Tabacman, H., 1984 Effect of crop level on growth, yield and wine quality of a high yielding Carignane vineyard. Am. J. Enol. Vitic. 35, 247-252.

Bravdo, B., Hepner, Y., Loinger, C., Cohen, S. \& Tabacman, H., 1985. Effect of crop level and crop load on growth, yield, must and wine composition, and quality of Cabernet Sauvignon. Am. J. Enol. Vitic. 36, 125-131.

Buttery, R.G.B., Seifert, R.M., Guadagni, D.G., Black, D.R. \& Ling, L.C., 1968. Characterization of some volatile constituents of carrots. J. Agric. Food Chem. 16, 1009-1015.

Buttery, R.G.B., Seifert, R.M., Ling, L.C., Soderstrom, E.L., Ogawa, J.M. \& Turnbaugh, J.G., 1982. Additional aroma components of honeydew melon. J. Agric. Food Chem. 30(6), 1208-1211.
Buttery, R.B.G., Turnbaugh, J.G. \& Ling, L.C., 1988. Contribution of volatiles to rice aroma. J. Agric. Food Chem. 36, 1006-1009.

Cadot, Y., Caillé, S., Samson, A., Barbeau, G. \& Cheynier, V., 2012. Sensory representation of typicality of Cabernet franc wines related to phenolic composition: Impact of ripening stage and maceration time. Anal. Chim. Acta 732, 91-99.

Casassa, L.F., Beaver, C.W., Mireles, M., Larsen, R.C., Hopfer, H., Heymann, H. \& Harbertson, J.F., 2013. Influence of fruit maturity, maceration length, and ethanol amount on chemical and sensory properties of Merlot wines. Am. J. Enol. Vitic. 64, 437-449.

Chapman, D.M., Matthews, M.A. \& Guinard, J.-X., 2004. Sensory attributes of Cabernet Sauvignon wines made from vines with different crop yields. Am. J. Enol. Vitic. 55, 325-334

Chkaiban, L., Botondi, R., Bellincontro, A., De Santis, D., Kefalas, P. \& Mencarelli, F., 2007. Influence of postharvest water stress on lipoxygenase and alcohol dehydrogenase activities, and on the composition of some volatile compounds of Gewürztraminer grapes dehydrated under controlled and uncontrolled thermohygrometric conditions. Austr. J. Grape Wine Res. $13,142-149$

Cordonnier, R.E. \& Bayonove, C.L., 1974. Mise en évidence dans la baie de raisin, variété Muscat d'Alexandrie, de monoterpènes liés, révélables par une ou plusieurs enzyme du fruit. C.R. Acad. Sci. Paris 278, 3387-3390.

Costantini, V., Bellincontro, A., De Santis, D., Botondi, R. \& Mencarelli, F., 2006. Metabolic changes of Malvasia grapes for wine production during postharvest drying. J. Agric. Food Chem. 54, 3334-3340.

Costantini, A., García-Moruno, E. \& Moreno-Arribas, M.V., 2009. Biochemical transformations produced by malolactic fermentation. In Moreno-Arribas, M.V. (ed.). Wine chemistry and biochemistry. Springer, New York. pp. $27-57$

Diago, M.P., Vilanova, M., Blanco, J.A. \& Tardaguila, J., 2010. Effects of mechanical thinning on fruit and wine composition and sensory attributes of Grenache and Tempranillo varieties (Vitis vinifera L.). Austral. J. Grape Wine Res. 16, 314-326.

Di Profio, F.A., Reynolds, A.G. \& Kasimos, A., 2011. Canopy management and enzyme impacts on Merlot, Cabernet franc, and Cabernet Sauvignon. II Wine composition and quality. Am. J. Enol. Vitic. 62, 152-168.

Dunlevy, J.D., Kalua, C., Keyzers, R. \& Boss, P.K., 2009 (2nd ed.). The production of flavour and aroma compounds in grape berries. In: RoubelakisAngelakis, K.A. (ed.). Grapevine molecular physiology and biotechnology Springer Science + Business Media, Berlin, Germany. pp. 293 - 340.

Dunlevy, J.D., Soole, K.L., Perkins, M.V., Nicholson, E.L., Maffei, S.M. \& Boss, P.K., 2013. Determining the methoxypyrazine biosynthesis variables affected by light exposure and crop level in Cabernet Sauvignon. Am. J. Enol. Vitic. 64, 450-458.

Escudero, A., Gogorza, B., Melus, M., Ortin, N., Cacho, J. \& Ferreira, V., 2004. Characterization of the aroma of a wine from Maccabeo. Key role played by compounds with low odor activity values. J. Agric. Food Chem. $52,3516-3524$

Etiévant, P.X.E., 1991. Wine. In: Maarse, H. (ed.). Volatile compounds in foods and beverages. Food Science and Technology, Dekker, New York. pp. $483-546$.

Ferreira, V., López, R. \& Cacho, J.F., 2000. Quantitative determination of the odorants of young red wines from different grape varieties. J. Sci. Food Agr. 80, 1659-1667.

Gamero, E., Moreno, D., Vilanova, M., Uriarte, D., Prieto, M.H. \& Esperanza Valdés, M., 2014. Effect of bunch thinning and water stress on chemical and sensory characteristics of Tempranillo wines. Aust. J. Grape Wine Res. 20, 394-400. 
Gunata, Y.Z., Bayonove, C.L., Baumes, R.L. \& Cordonnier, R.E., 1985. The aroma of grapes. I. Extraction and determination of free and glycosidically bound fractions of some grape aroma components. J. Chromatography A 331, 83-90.

Guth, H., 1997. Identification of character impact odorants of different white wine varieties. J. Agric. Food Chem. 45, 3022-3026.

Kays, S.J., 1997. Stress in harvested products. In: Kays, S.J. (ed.). Postharvest physiology in perishable plant products. Exon Press, Athens, GA. pp. $335-408$.

Keller, M., Mills, L.J., Wample, R.L. \& Spayd, S.E., 2005. Cluster thinning effects on three deficit-irrigated Vitis vinifera cultivars. Am. J. Enol. Vitic. $56,91-103$.

Keller, M., Smithyman R.P. \& Mills L.J. 2008. Interactive effects of deficit irrigation and crop load on Cabernet Sauvignon in an arid climate. Am. J. Enol. Vitic. 59, 221-234.

King, P.D., McClellan D.J. \& Smart R.E., 2012. Effect of severity of leaf and crop removal on grape and wine composition of Merlot vines in Hawke's Bay vineyards. Am. J. Enol. Vitic. 63, 500-507.

Kliewer, W.M. \& Dokoozlian N.K., 2005. Leaf area/crop weight ratios of grapevines: Influence on fruit composition and wine quality. Am. J. Enol. Vitic. 56, 170-181

Laffort, 2013. L'Oenologie par Nature [Online]: https://www.laffort.com/ en/products/enzymes [accessed on 23 May 2013]

Lefebvre, R., Reynolds, A.G. \& Di Profio, F.A., 2015. Crop load and harvest date have minimal impact on bud cold hardiness and cane carbohydrate levels of four grapevine cultivars. Vitis 54, 189-193.

Marais, J., 1983. Terpenes in the aroma of grapes and wines: A review. S. Afr. J. Enol. Vitic. 4, 49-60.

Marais, J., 1987. Terpene concentrations and wine quality of Vitis vinifera L. cv. Gewurztraminer as affected by grape maturity and cellar practices. Vitis 26, 231-245.

Marais, J. \& Van Wyk, C.J., 1986. Effect of grape maturity and juice treatments on terpene concentrations and wine quality of Vitis vinifera $\mathrm{L}$. cv. Weisser Riesling and Bukettraube. S. Afr. J. Enol. Vitic. 7, 26-35.

Mateo, J.J. \& Jimenez, M., 2000. Review. Monoterpenes in grape juice and wines. J. Chromatography A 881, 557-567.

Matthews, M.A. \& Nuzzo, V. 2007. Berry size and yield paradigms on grapes and wine quality. Acta Hort. 754, 423-435.

Meyers, J.M., Sacks, G.L. \& Vanden Heuvel, J.E., 2013. Glycosylated aroma compound responses in 'Riesling' wine grapes to cluster exposure and vine yield. Horttechnol. 23, 581-588.

Michlmayr, H., Nauer, S., Brandes, W., Schümann, C., Kulbe K.D, Del Hierro, A.M. \& Eder, R., 2012. Release of wine monoterpenes from natural precursors by glycosidases from Oenococcus oeni. Food Chem. 135, 80-87.

Moio, L., Ugliano, M., Gambuti, A., Genovese, A. \& Piombino, P., 2004. Influence of clarification treatment on concentrations of selected free varietal aroma compounds and glycoconjugates in Falanghina (Vitis vinifera L.) must and wine. Am. J. Enol. Vitic. 55, 7-12.

Moreno, J.J., Cerpa-Calderón, F., Cohen, S.D., Fang, Y., Qian, M. \& Kennedy, J.A., 2008. Effect of postharvest dehydration on the composition of Pinot noir grapes "Vitis vinifera" L. and wine. Food Chem. 109, 755-762.
Moreno Luna, L.H., Reynolds, A.G. \& Di Profio, F.A., 2017. Crop level and harvest date impact composition of four Ontario wine grape cultivars. I. Yield, fruit, and wine composition. Am. J. Enol. Vitic. 68, 431-446.

Naor, A., Gal, Y. \& Bravdo, B., 2002. Shoot and cluster thinning influence vegetative growth, fruit yield, and wine quality of Sauvignon blanc grapevines. J. Am. Soc. Hortic. Sci. 127, 628-634.

Ough, C. \& Nagaoka, R., 1984. Effect of cluster thinning and vineyard yields on grape and wine composition and wine quality of Cabernet Sauvignon. Am. J. Enol. Vitic. 35, 30-34.

Preszler, T., Schmit, T.M. \& Vanden Heuvel, J.E., 2013. Cluster thinning reduces the economic sustainability of Riesling production. Am. J. Enol. Vitic. 64, 333-341.

Rapp, A. \& Mandery, H., 1986. Wine aroma. Experientia 42, 873-884.

Reynolds, A.G. \& Wardle, D.A., 1989. Impact of several canopy manipulation practices on growth, yield, fruit composition, and wine quality of Gewurztraminer. Am. J. Enol. Vitic. 40, 121-129.

Reynolds, A.G., Edwards, C.G., Wardle, D.A., Webster, D.R. \& Dever, M.C., 1994. Shoot density affects 'Riesling' grapevines. II. Wine composition and sensory response. J. Am. Soc. Hort. Sci. 119, 881-892.

Reynolds, A.G., Schlosser, J., Sorokowsky, D., Roberts, R., Willwerth, J.J. \& De Savigny, C., 2007. Magnitude of viticultural and enological effects. II. Relative impacts of cluster thinning and yeast strain on composition and sensory attributes of Chardonnay musqué. Am. J. Enol. Vitic. 58, 25-41.

Reynolds, A.G., Yerle, S., Watson, B.T., Price, S.F. \& Wardle, D.A., 1996. Fruit environment and crop level effects on Pinot noir. III. Composition and descriptive analysis of Oregon and British Columbia wines. Am. J. Enol. Vitic. 47, 329-339.

Robinson, A.L., Boss, P.K., Solomon, P.S., Trengove, R.D., Heymann, H. \& Ebeler, S.E., 2014. Origins of grape and wine aroma. Part 1. Chemical components and viticultural impacts. Am. J. Enol. Vitic. 65, 1-24.

Ruth, J.H., 1986. Odor thresholds and irritation levels of several chemical substances: A review. Am. Ind. Hygiene Assoc. J. 47, 142-151.

Schwab, W., Davidovich-Rikanati, R. \& Lewinsohn, E., 2008. Biosynthesis of plant-derived flavor compounds. Plant J. 54, 712-732.

Sponholz, W., 1993. Wine spoilage by microorganisms. In: Fleet, G. (ed.). Wine microbiology and biotechnology. Harwood Academic Publisher, Chur, Switzerland. pp. $395-420$.

Sun, Q., Sacks, G.L., Lerch, S.D. \& Vanden Heuvel, J.E., 2011. Impact of shoot thinning and harvest date on yield components, fruit composition, and wine quality of Marechal Foch. Am. J. Enol. Vitic. 62, 32-41.

Takeoka, G.R., Flath R.A., Mon, T.R., Teranishi, R. \& Guentert, M., 1990. Volatile constituents of apricot (Prunus armeniaca). J. Agric. Food Chem. $38,471-477$.

Zamboni, A., Minoia, L., Ferrarini, A., Tornielli, G.B., Zago, E., Delledonne, M. \& Pezzotti, M., 2008. Molecular analysis of post-harvest withering in grape by AFLP transcriptional profiling. J. Exp. Bot. 59, 4145-4159.

Zamora, F., 2009. Biochemistry of alcoholic fermentation. In: MorenoArribas, M.V. (ed.). Wine chemistry and biochemistry. Springer, New York. pp. $3-26$.

Zoecklein, B.W., Williams, J. \& Duncan, S., 2010. Effect of sour rot on the composition of White Riesling (Vitis vinifera L.) grapes. Small Fruit Rev. $1,63-77$. 